\title{
Aménagement forestier et lutte contre la pauvreté au Burkina Faso
}

Boukary Ouedraogo

\section{OpenEdition}

Journals

Édition électronique

URL : http://journals.openedition.org/developpementdurable/8215

DOI : 10.4000/developpementdurable.8215

ISSN : 1772-9971

\section{Éditeur}

Association DD\&T

\section{Référence électronique}

Boukary Ouedraogo, «Aménagement forestier et lutte contre la pauvreté au Burkina Faso »,

Développement durable et territoires [En ligne], Varia (2004-2010), mis en ligne le 01 septembre 2009,

consulté le 30 avril 2019. URL : http://journals.openedition.org/developpementdurable/8215 ; DOI :

10.4000/developpementdurable.8215

Ce document a été généré automatiquement le 30 avril 2019.

\section{(cc) (i) \&)}

Développement Durable et Territoires est mis à disposition selon les termes de la licence Creative

Commons Attribution - Pas d'Utilisation Commerciale 4.0 International. 


\title{
Aménagement forestier et lutte contre la pauvreté au Burkina Faso
}

\author{
Boukary Ouedraogo
}

1 La gestion des ressources forestières en Afrique Occidentale remonte à la période coloniale avec la constitution d'un domaine forestier classé depuis 1933. La gestion des ressources naturelles est un système visant à contrôler l'usage des ressources de manière à éviter le gaspillage et à optimiser cet usage pour en tirer le meilleur profit possible (Collins, 1992). Les politiques forestières au Burkina peuvent être scindées en deux périodes caractérisées par des méthodes de gestion forestière bien typées.

2 La période des années 60 (période « des indépendances »), caractérisée par des politiques forestières héritées de la colonisation, où des politiques de conservation ont été édictées par l'État, prévoyant l'exclusion totale des populations riveraines des zones mises en réserve, tandis que la gestion des zones exploitées était planifiée de façon centralisée (Ribot, 2001). Ces politiques forestières centralisées et répressives vont vite révéler leurs insuffisances pour la sauvegarde et l'exploitation durable des ressources forestières. Ainsi, les populations locales dont la vie allait être affectée par les interventions prévues n'étaient ni prises en compte, ni consultées, ni informées, mais bien réquisitionnées pour satisfaire aux besoins de main d'oeuvre de l'exploitation des forêts (Delnooz, 2003). Il en résulta des conflits et confrontations entre les différentes parties. Ces échecs dans la gestion des ressources forestières, amplifiés par la crise du bois-énergie dans la capitale du pays et les sécheresses consécutives à la fin des années 70, vont conduire les pouvoirs publics à repenser les politiques forestières, adoptant ainsi de nouvelles approches socioculturelles basées sur la participation active et volontaire des populations locales comme facteur central de démarrage d'une exploitation soutenue de ces ressources. Cette période caractérisée par une gestion participative des forêts s'installera définitivement en 1986 sous la forme d'un projet pilote nommé « Aménagement des Forêts Naturelles » visant à approvisionner les populations en bois-énergie et à sauvegarder l'environnement (Kaboré, 2004).

3 Selon la $\mathrm{FAO}^{1}$, dans la plupart des débats sur la foresterie et le développement, qui se déroulent dans les instances internationales, on cherche à identifier les moyens 
d'intégrer les forêts et la foresterie dans les Objectifs de Développement pour le Millénaire des Nations Unies, visant à réduire de moitié la pauvreté et l'insécurité alimentaire d'ici 2015. En effet, ddepuis le premier examen de ses politiques commerciales en 1998, le Burkina Faso a changé son programme pour le développement du pays, ayant adopté en 2000 un Cadre Stratégique de Lutte contre la Pauvreté (CSLP), dont la première révision a eu lieu en septembre 2003. C'est dans cette perspective que s'inscrit ce papier, qui, loin d'occulter l'impact de l'aménagement forestier sur la durabilité des ressources forestières, tente d'appréhender ses effets sur les conditions de vie des bûcherons et des populations riveraines des forêts aménagées. "L'aménagement forestier au Burkina Faso a-t-il contribué à réduire la pauvreté au sein des populations riveraines des forêts aménagées?» Cette question est d'un intérêt public avéré d'autant plus que parmi les agriculteurs vivriers du pays dont font partie les exploitants forestiers, l'indice de pauvreté s'établit à 52,3\% avec une contribution à l'indice global de pauvreté de 77,1\% (INSD, 2003). Ce niveau de pauvreté s'amplifie avec les aléas climatiques qui hypothèquent les campagnes agricoles, orientant pendant ces années de sécheresse les paysans vers une exploitation anarchique du potentiel forestier. C'est en cela que l'aménagement forestier s'impose comme instrument devant intégrer dans les politiques d'aménagement forestier, les Objectifs de Développement pour le Millénaire.

Des auteurs comme Ribot (1995), Foley et al. (1997; 2002), Hautdidier et al. (2004), Atchoumgaï (2003), Hautdidier et Gautier (2005) et Ouédraogo (2007) ont déjà investigué sur les marchés ruraux du bois d'énergie et le contrôle des forêts locales en Afrique au Sud du Sahara et plus particulièrement au Burkina Faso, au Mali et au Niger. Ces auteurs se sont interrogés sur les effets de la gestion décentralisée et participative des forêts sur les exploitants $\mathrm{du}$ bois d'énergie, et comment ces réformes ont bénéficié économiquement et socialement à ces producteurs locaux. Hautdidier et Gautier (2005) concluent que la création et l'installation des marchés ruraux du bois de chauffe ont eu des effets significatifs au plan local et que la coupe de bois et la construction de fours ont une influence croissante car elles sont considérées comme une stratégie intéressante de diversification pour la survie des ménages ruraux en élevant leur sécurité alimentaire ; ils ajoutent que, sur le plan économique, ces activités relatives au bois de feu génèrent des revenus non seulement chez les ménages et les individus, mais aussi à l'échelle du village riverain à travers la construction et la restauration des infrastructures communautaires. Ces études utilisent la méthode "avant-après » pour appréhender l'effet de la gestion décentralisée et participative sur les conditions de vie des ménages ruraux, méthode qui est fondée sur l'évaluation de la perception des bûcherons par rapport à l'évolution de variables ciblées. Ouédraogo (2007) utilise une approche filière pour construire la structure des prix du bois-énergie exploité dans les zones forestières aménagées du Burkina et commercialisé à Ouagadougou, et en déduit une clé de répartition des bénéfices de la filière entre les différents intervenants. Les résultats de cette étude appliquée aux forêts aménagées du Burkina, révèlent que les bûcherons bénéficient moins de l'exploitation de la ressource ligneuse que les autres intervenants de l'aval de cette filière, et se positionnent au troisième rang après les grossistes-transporteurs premiers bénéficiaires et les détaillants du bois-énergie (Op. cit., 2007 : 86). Les indicateurs utilisés par ces études ne permettent pas d'appréhender la pauvreté parmi les populations cibles. C'est pourquoi dans la présente étude, nous privilégierons d'autres indicateurs plus adaptés à l'étude de la pauvreté. 
Cet article propose d'évaluer la contribution de l'aménagement forestier à la réduction de la pauvreté. Les trois principales hypothèses qui guident notre réflexion sont les

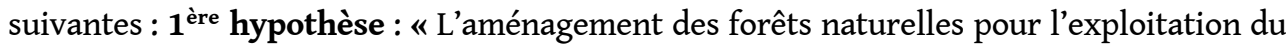
bois-énergie a contribué à générer de nouvelles sources de revenus pour les populations riveraines de ces forêts ». Les sources initiales de revenus exploitants forestiers avant aménagement forestier étant essentiellement constitués des revenus de surplus agricoles, des ventes de produits forestiers non ligneux (fruits et légumes sauvages). $2^{\text {ème }}$ hypothèse : « Les revenus tirés de l'exploitation du bois-énergie sont prédominants dans la structure des revenus des bûcherons dans les villages riverains des zones forestières aménagées du Burkina Faso ».Cette hypothèse est d'autant plus intéressante qu'elle met en évidence l'importance des activités forestières dans l'amélioration des conditions de vie des populations riveraines des forêts aménagées. $3^{\text {ème }}$ hypothèse $:$ La majeure partie des bûcherons des zones forestières aménagées vivent au-dessus du seuil de pauvreté du Burkina Faso.

6 Ces hypothèses nous amèneront respectivement à présenter les politiques d'aménagement forestier au Burkina Faso, à explorer la littérature sur la notion de pauvreté et les instruments de sa mesure, à étudier la structure des revenus des bûcherons dans les zones forestières aménagées et à calculer des indices appropriés de pauvreté en vue d'analyser la pauvreté parmi les bûcherons de ces zones forestières. Avant d'esquisser une étude des éléments conceptuels et des mesures de la pauvreté, il apparaît nécessaire de décrire les sources statistiques qui vont être utilisées et de présenter la population cible.

\section{Sources statistiques et renseignements sur les exploitants forestiers}

7 Les données statistiques utilisées dans cette étude sont issues de larges enquêtes que nous avons élaborées et réalisées sous financement de USAID et de l'assistance technique du Centre International de Recherche en Foresterie (CIFOR) en mai-juin 2005, sur l'ensemble des zones forestières aménagées de l'ex « Projet Aménagement des Forêts Naturelles » au Burkina Faso. Ces enquêtes ont couvert 24 villages situés entre $70 \mathrm{~km}$ et $250 \mathrm{~km}$ de la ville de Ouagadougou de 8 chantiers aménagés. Les tailles respectives des populations cibles de ces enquêtes ont été : 355 bûcherons et charbonniers, 8 directeurs techniques des chantiers aménagés, et 16 bureaux exécutifs des groupements villageois de gestion forestière et 4 bureaux exécutifs des unions des groupements de gestion forestière. La répartition de ces producteurs selon le chantier et la province est donnée par le tableau $n$ $\circ 1$ ci-dessous. 
Tableau 1 : Répartition des producteurs selon le chantier forestier et la province de localisation

\begin{tabular}{lcccccc}
\hline \multirow{2}{*}{$\begin{array}{l}\text { Noms des chantiers } \\
\text { aménagés }\end{array}$} & Ziro & Sissili & Sanguié & Bazega & Kadiogo & Total \\
\hline Cassou & 45 & 0 & 0 & 0 & 0 & 45 \\
Silly-Pouni-zazwara & 0 & 52 & 31 & 0 & 0 & 83 \\
Sud Ouest sissili & 0 & 48 & 0 & 0 & 0 & 48 \\
Sapoui Bieha & 46 & 0 & 0 & 0 & 0 & 46 \\
Nazinon & 43 & 0 & 0 & 0 & 0 & 43 \\
Nakambé & 0 & 0 & 0 & 32 & 0 & 32 \\
Gonsé & 0 & 0 & 0 & 0 & 18 & 18 \\
Bougnounou & 0 & 40 & 0 & 0 & 0 & 40 \\
\hline Total & $\mathbf{1 3 4}$ & $\mathbf{1 4 0}$ & $\mathbf{3 1}$ & $\mathbf{3 2}$ & $\mathbf{1 8}$ & $\mathbf{3 5 5}$ \\
\hline
\end{tabular}

Source : Analyse des données d'enquêtes bûcherons (mai-juin 2005).

8 Dans l'ensemble, les 355 producteurs de bois-énergie sont visités et sondés dans 8 chantiers aménagés géographiquement situés dans les provinces de la Sissili, du Sanguié, du Bazega, du Kadiogo et du Ziro. Ces chantiers sont autonomes et gérés par des groupements villageois appuyés par des ingénieurs forestiers. Ces chantiers forestiers regroupent chacun plusieurs villages riverains. Le chantier de Gonsé est inclus dans l'échantillon pour sa très grande proximité de la ville de Ouagadougou, bien qu'aujourd'hui, la fermeture du projet «Gestion Forestière Intégrée » qui en avait la charge laisse entrevoir une extinction de la couverture végétale de cette forêt. Toutefois, la forêt de Goinsé est exclue de nos analyses car les activités d'activités d'aménagement y ont pris fin depuis 2001. Un retraitement des données d'enquêtes a permis de retenir 300 exploitants pour les analyses, certaines observations ayant simplement été éliminées pour des raisons de cohérence des données recueillies.

9 Les bûcherons des zones forestières aménagées du Burkina sont constitués essentiellement par les populations riveraines des forêts, qui décident d'adhérer au groupement villageois de gestion forestière, s'engagent à respecter les statuts et règlements intérieurs et à prendre activement part aux activités d'exploitation forestière. Parmi les bûcherons enquêtés, 4,5 \% sont des femmes et 95,5\% d'hommes. 76,2 \% d'entre eux n'avaient aucun niveau d'instruction formelle, $19,3 \%$ avaient eu un niveau d'éducation primaire et seulement $4,5 \%$ avaient pu suivre des études secondaires.

\section{Les politiques d'aménagement forestier au Burkina Faso}

10 Cette section présente les politiques d'aménagement forestier du Burkina en mettant en exergue les cadres juridique et institutionnel de ces politiques ainsi que les éléments de gestion sur la base desquels ces politiques sont mises en œuvre.

\subsection{Les cadres juridique et institutionnel}

11 L'aménagement forestier au Burkina Faso se fonde sur des dispositions légales, institutionnelles, stratégiques et techniques relatives aux préoccupations économiques et sociales dont la conjonction devrait assurer une durabilité dans la gestion des ressources forestières prônée par la politique forestière nationale (MET, 2002). Les principales dispositions orientant l'aménagement des forêts sont: la Réforme Agraire et Foncière 
(RAF), le code de l'environnement, le code Forestier, les Textes d'Orientations de la Décentralisation (TOD), la Politique Forestière Nationale et le Programme National d'Aménagement des Forêts (PNAF) (Sawadogo, 2006, p. 6).

L'aménagement forestier, en tant qu'acte fondamental de la gestion forestière, est au centre de nombreux débats au sein de la communauté scientifique et forestière (Peyre, 1999 ; Boutefeu, 2005). La notion d'aménagement forestier connaît plusieurs définitions, quelques unes bénéficient d'un large consensus dont celle de Dubourdieu (1997) la définissant comme: "étude et document sur lesquels s'appuie la gestion durable d'une forêt à partir d'analyses du milieu naturel et du contexte économique et social, l'aménagement fixe les objectifs et détermine l'ensemble des interventions souhaitables (coupes, travaux...) pendant une durée de 10 à 25 ans ». De ce point de vue, l'expression "Aménagement des forêts » recouvre tous les aspects administratifs, économiques, juridiques, sociaux, techniques et scientifiques de la conservation et de l'utilisation des forêts (FAO, 1996). Il s'agit donc de l'application de la foresterie à une aire géographique donnée en vue d'assurer aux communautés les avantages qui en découlent, et aux gestionnaires un revenu optimal et soutenu. Ainsi selon Boutefeu (2005), "L'aménagement peut être considéré comme la cheville ouvrière du forestier, l'outil grâce auquel il définit et applique une politique. C'est par cet acte qu'il détermine les objectifs et les moyens de sa gestion. Ces derniers sont consignés dans un document de référence appelé plan l'aménagement $»$. La zone d'aménagement correspond à l'endroit où est pratiqué l'aménagement. L'exploitation du bois dans les zones aménagées se fait suivant les normes sylvicoles. Dans l'expérience burkinabé d'aménagement forestier, les bûcherons sont formés en techniques sylvicoles, notamment dans les techniques de coupe, et sont encadrés par un ingénieur forestier appelé Directeur technique, qui assure la gestion technique de l'exploitation durable de la forêt. Le modèle de gestion adopté est la gestion participative qui "est un partenariat où deux ou plusieurs parties prenantes négocient ensemble, s'entendent et exécutent des fonctions, des bénéfices et des responsabilités liés à un territoire particulier ou à un ensemble de ressources naturelles » (Gray, 1998).

13 Au niveau de chaque chantier d'aménagement forestier (CAF), les populations locales partenaires sont organisées en Groupements Villageois de Gestion Forestière (GVGF) formés aux techniques de gestion des ressources forestières, qui ont en charge la gestion des ressources forestières et la promotion du développement local. Ces groupements villageois constituent des groupements associatifs ou coopératifs. Selon le Bureau International du Travail, la coopérative se définit comme » une association de personnes qui se sont volontairement groupées pour atteindre un but commun par la constitution d'une entreprise dirigée démocratiquement, en fournissant une quote-part équitable du capital nécessaire et en acceptant une juste participation aux risques et aux fruits de cette entreprise, au fonctionnement de laquelle les membres participent activement ». Ces GVGF sont aussi chargés de la protection des Unités d'Aménagement Forestier (UAF) contre toute forme d'occupation non conforme à l'esprit de l'aménagement dans les villages associés à l'aménagement. Pour plus de crédibilité, les GVGF ont mis en place, des organisations faîtières: les Unions des Groupements Villageois de Gestion Forestière (UGVGF), celles-ci se regroupant pour former une Fédération des Unions des Groupements Villageois de Gestion Forestière (FUGVGF). L'Etat qui reste le propriétaire des terres, concède aux UGVGF l'autonomie de la gestion de la zone forestière consignée dans un cahier des charges. forestier. L'exploitation forestière s'y réalise selon un plan d'aménagement, un cahier de 
charge et un contrat de gestion. Le contrat de gestion stipule que: "L'Union des Groupements de Gestion Forestière du Chantier d'Aménagement Forestier jouit d'un droit d'usufruit sur les ressources forestières du chantier en question durant la période couverte par le plan d'aménagement forestier ", la terre appartenant à l'Etat selon la Réforme Agraire Foncière (RAF). Selon le cahier des charges régissant la concession du Chantier d'Aménagement Forestier (CAF), « Le CAF est un ensemble constitué d'un massif forestier mis en valeur et soumis à un plan de gestion à travers : une composante sociale qui est l'Union des Groupements de Gestion Forestière, un Conseil de gestion, un Comité de contrôle interne, une Commission de contrôle, une Direction Technique et une ressources financière substantielle qui est le Fonds d'Aménagement Forestier. Les ressources du CAF sont constituées par : les parts sociales des groupements membres, les cotisations prévues par le Règlement Intérieur, la contribution en équipement et infrastructures des donateurs publics et privés, les emprunts auprès des groupements, autres sociétés coopératives, banques, organismes de crédits publics ou privés ou tout autre institution d'assistance régulièrement reconnue par l'Etat ». Le Cahier de Charges du CAF notifie que l'objectif de la gestion est de contribuer à l'exploitation rationnelle et durable des ressources forestières par les communautés villageoises riveraines organisées en Groupements de Gestion Forestière (GVGF) en vue de ravitailler les grands centres urbains et les autres localités en produits forestiers. Cet objectif doit contribuer par ailleurs à la gestion optimale et durable de l'ensemble des productions agricoles, pastorales et forestières.

\subsection{Les éléments de gestion des forêts naturelles}

\section{Les Activités et les techniques d'aménagement utilisées}

Les activités d'aménagement et les techniques utilisées concernent respectivement la coupe de bois, l'enrichissement par semis direct, la mise en repos des parcelles exploitées, les feux précoces et la commercialisation du bois exploité (Sawadogo, 2006 : 11-14).

La coupe de bois concerne aussi bien le bois mort que le bois vert. L'exploitation de bois mort se fait en toute saison tandis que celle du bois vert comporte plusieurs étapes selon un calendrier bien établi :d'une part, la délimitation des parcelles de coupe, qui se fait chaque année dès la fin de la saison pluvieuse, avec une identification et une programmation des parcelles à exploiter par le Directeur technique du chantier, qui les indique aux GVGF ; d'autre part, la coupe de bois vert se fait entre le mois de janvier et celui de mars chaque année, et dirigée par un moniteur qualifié, qui marque les individus devant être coupés (martelage). Les critères de coupe appliqués sont respectivement la densité du peuplement, l'exploitation étant interdite dans les sites présentant moins de 200 pieds/ha ; l'état sanitaire, qui privilégie prioritairement la coupe des arbres malades ou malformés; les considérations écologiques, qui épargnent les espèces protégées ou rares et les semenciers; les dimensions de commercialisation qui orientent l'exploitation sur les arbres dont le diamètre à hauteur de poitrine est comprise entre 10 et $25 \mathrm{~cm}$. La technique d'abattage qui est celle à entaille double, permet d'orienter la chute de l'arbre et de laisser une souche en biseau réduisant la pourriture de la souche par stagnation des eaux de pluies ; la hauteur de coupe fixée à $15 \mathrm{~cm}$ maximum pour permettre l'émergence de rejets de souche le plus proche du sol ; le type de coupe consiste en une coupe sélective avec un prélèvement maximal de $50 \%$ du volume commercialisable sur pied et constitue la prescription sur tous les chantiers d'aménagement. La rotation qui définit l'intervalle 
de temps séparant deux coupes successives d'une parcelle, celle-ci variant de 15 ans à 20 ans selon le CAF.

17 L'enrichissement par semis direct consistant en des mesures de restauration ou de réhabilitation des parcelles exploitées. Les semis directs comme mode de régénération sont appliqués dans les CAF sous deux formes : le semi d'afforestation qui est exécuté de manière systématique sur toutes les zones nues ainsi que les champs et jachères jeunes ; et le semi d'enrichissement qui se réalise sur les parcelles de coupe annuelle juste après l'exploitation. Les opérations les plus importantes liées au semis d'enrichissement sont le choix des espèces, la récolte et la conservation des semences et l'opération de semis proprement dite.

a mise en repos des parcelles exploitées préconise l'interdiction du feu et du pâturage sur les parcelles exploitées pendant une période de 3 à 5 ans pour permettre au semis et rejets de croître afin de s'affranchir du feu et de la dent du bétail. Les membres des GVGF sont chargés de veiller au respect de ces prescriptions par la surveillance.

Les feux précoces sont pratiqués annuellement de manière systématique sur l'ensemble des unités entre Octobre et Décembre, exception faite des parcelles nouvellement exploitées. Il s'agit de mettre le feu pendant que la strate herbacée est toujours relativement humide.

La commercialisation consiste à empiler le bois exploité en doubles stères le long des voies d'accès aux parcelles, et à le vendre aux grossistes-transporteurs par l'intermédiaire du commis.

21 Ainsi, le critère de la durabilité de la forêt est celui le plus important dans la mise en œuvre de l'aménagement forestier au Burkina. Aujourd'hui, près de 667600 hectares de forêts sont aménagés dont $50 \%$ sont autonomes et gérés par les unions et groupement de gestion forestières; ils comptent près de 8 Unions de Gestion Forestière officiellement reconnues qui regroupent plus de 250 Groupements Villageois de Gestion Forestière. Il demeure 202400 hectares en instance d'aménagement.

\section{Les instruments économiques de gestion de l'exploitation forestière}

Le tableau 2 permet d'examiner les différents instruments économiques de gestion du patrimoine forestier en vigueur en amont dans les zones forestières aménagées. Ce tableau indique la destination des recettes issues de ces instruments et les fonctions qu'ils assurent. S'agissant de la gestion des recettes générées par la vente des produits, le fondus du chargement du bois est réglé au niveau du commis de commercialisation à raison de $2200 \mathrm{~F}$ CFA par stère de bois et est reparti conformément à une clé de répartition dont les rubriques sont définies dans le même tableau.

Tableau 2 : Instruments de gestion de forestières/structure des prix en amont

\begin{tabular}{|l|c|c|c|}
\hline $\begin{array}{c}\text { Libellés des postes de la } \\
\text { structure des prix }\end{array}$ & $\begin{array}{c}\text { Valeur en FCFA } \\
\text { /Stère }\end{array}$ & Destination des recettes & Fonctions \\
\hline Taxe forestière & 300 & $\begin{array}{c}50 \% \text { aux collectivités } \\
\text { décentralisées et } 50 \% \text { au } \\
\text { trésor public }\end{array}$ & $\begin{array}{c}\text { Fonction de trésorerie / rente de } \\
\text { propriété. }\end{array}$ \\
\hline Redevance forestière & 600 & Le fonds d'aménagement & $\begin{array}{c}\text { Fonction de reconstitution et/ou de } \\
\text { conservation de la ressource }\end{array}$ \\
\hline Fonds de roulement ${ }^{2}$ & 200 & Investissement collectif & $\begin{array}{c}\text { Augmenter le bien-être collectif des } \\
\text { riverains }\end{array}$ \\
\hline Rémunération bûcheron & 1100 & & \\
\hline $\begin{array}{l}\text { Prix de cession au } \\
\text { grossiste }\end{array}$ & 2200 & & \\
\hline
\end{tabular}

Sources : Ouédraogo (2002, p. 285) 
orestière, communément appelée permis de coupe est un prix fixé à 300 FCFA par stère de bois coupé en forêt ou en toute zone boisée non privée. Pour Barde (1992), « la taxe se définit comme un prélèvement obligatoire de l'État, effectué sans contrepartie directe. Les recettes issues de la taxe sont versées au budget général de l'État sans affectation particulière. La taxe est un paiement effectué sur chaque unité de ressource prélevée et le taux de taxe représente en quelque sorte le prix de l'utilisation de celle-ci ». C'est une rente de propriété pour l'État. La taxe forestière a deux fonctions. D'une part, la taxe peut permettre de saisir la quantité de bois coupé pendant une période de temps donnée, si les moyens de contrôle et d'application de ces instruments sont disponibles et fonctionnent correctement ; d'autre part, la taxe peut être une source de revenu pour le Trésor Public.

Thiam (1993) définit la redevance comme la contrepartie monétaire d'un prélèvement sur une ressource renouvelable non renouvelée (ou renouvelée mais insuffisamment renouvelée). Elle renvoie à la notion de coût de reconstitution de la ressource. Pour Barde (1992), » la redevance se définit comme un prélèvement comportant une contrepartie et les recettes issues de celle-ci sont affectées à des fins spécifiques de lutte contre la dégradation de l'environnement ; la redevance est un paiement effectué sur chaque unité ressource prélevée.»

d'aménagement fixé à 600 FCFA le stère dans les chantiers forestiers aménagés répond à la définition de la redevance au regard de la destination de cette somme dont la totalité devrait servir à reconstituer les forêts exploitées. Cette appellation du fonds d'aménagement tient au caractère environnementaliste de la recette issue de cet instrument : financer la régénération et la protection des forêts. Il faut également noter que la redevance permet de saisir les quantités prélevées d'une ressource ou le niveau d'une pollution quelconque. Taxes et redevances ont une fonction d'internalisation des coûts sociaux liés à l'usage de ces ressources, et concourent ensemble dans des conditions correctes d'application et de suivi, à réduire les coupes anarchiques des ressources ligneuses et donc à leur bonne gestion. Elles ont en outre une fonction de contrôle des volumes de bois coupé et approvisionné aux ménages et sont destinées à l'aménagement des zones forestières.

\section{La pauvreté : éléments conceptuels et mesures}

Comme le note Dubois (1998:1), face à la pauvreté, et avant toute intervention visant à la réduire, on se pose généralement deux grandes questions: "- La première, de source conceptuelle, consiste à déterminer ce qu'est la pauvreté, ce qui, en termes opérationnels, peut se traduire par: à partir de quand peut-on considérer que l'on est pauvre? Ceci amène alors à distinguer diverses formes de pauvreté: pauvreté monétaire, pauvreté de conditions de vie et pauvreté de potentialités. La deuxième question, plus méthodologique, concerne la façon d'appréhender et de mesurer ces différentes formes. Elle se déduit naturellement de la question précédente et implique le recours à des méthodes particulières et, dans certains cas, à des instruments spécifiques. " 


\subsection{Une diversité des formes de pauvreté}

27 On peut distinguer à travers la littérature opérationnelle actuelle, trois grandes formes de pauvreté. La première forme de pauvreté, celle monétaire « ou de revenu », résulte d'une insuffisance de ressources et qui se traduit par une consommation insuffisante. La deuxième forme dite pauvreté des conditions de vie ou « d'existence » (Herpin et Verger, 1997), fait que l'on a du mal à satisfaire un certain nombre de besoins fondamentaux. Enfin, la troisième forme dite pauvreté de potentialités ou de «capacités ", fait que l'on ne peut engendrer le capital minimal (humain, social, physique, etc.) qu'il est nécessaire de posséder pour vivre, ou fonctionner, normalement dans une société donnée. Cette dernière notion récemment développée (Sen, 1993) fournit la justification théorique aux rapports annuels sur le développement humain publiés actuellement dans nombre de pays en développement, avec l'appui du Programme des Nations Unies pour le développement (PNUD).

Pour chacune de ces trois approches, il existe des justifications théoriques différentes en économie. Cependant, les différences théoriques entre ces approches s'estompent au fur et à mesure que progresse la connaissance du phénomène et que l'on assiste à une interaction entre les différents raisonnements (Dubois, 1998).

29 L'approche monétaire demeure rattachée à l'optique utilitariste et à l'économie du bienêtre, faisant toujours référence à la théorie de l'optimum général. Cependant l'intégration du capital humain (Becker, 1964 ; Schultz, 1961; Lucas 1988), des interactions sociales (Becker, 1974), puis, plus généralement, du capital social (Grootaert, 1997) tend à en élargir la vision.

L'approche en termes de conditions de vie s'appuyant sur la théorie des besoins essentiels, utilise le principe des lois d'Engel pour l'étude de la consommation alimentaire et fait implicitement appel aux notions de capital humain et social. Cette approche met l'accent sur les conditions de vie dans leur sens le plus large allant d'un manque de produits indispensables à l'origine, puis de biens essentiels (alimentation, habillement), à la difficulté d'accès à certains services (éducation, santé) et, maintenant, à la dégradation du lien social. Dans tous les cas la pauvreté se traduit alors par un manque dans certains aspects des conditions de vie.

31 L'approche en termes de potentialités va encore plus loin dans la même démarche faisant référence aux aspects de droits et d'obligation, de responsabilité intergénérationnelle, etc. Dans une telle optique, on peut aussi considérer la pauvreté non seulement comme un manque particulier (aspect conditions de vie), mais aussi comme l'impossibilité de réaliser à terme une potentialité qui aiderait à mieux fonctionner dans la vie. Cela peut notamment résulter d'une privation de droits ou de conflits intergénérationnels. Le fait d'introduire cette distinction dans l'analyse de la pauvreté, résulte de la volonté de mieux expliquer le mécanisme qui engendre la pauvreté en remontant à ses sources. Il s'agit de comprendre les comportements qui, dans une situation de pauvreté à un moment donné, ont créé les causes de pauvreté du futur. On retrouve, sous-jacent, la distinction entre pauvreté en amont et pauvreté en aval.

32 La différence entre ces trois approches réside dans la façon dont sont perçus et analysés les mécanismes économiques, en les considérant plus ou moins fortement enchâssés dans une réalité socio-économique (et temporelle) donnée qui accroît ou diminue les échecs du marché, pris comme norme de référence universelle (Dubois, 1998). Les analyses de 
Dubois (1998) montrent la difficulté à distinguer clairement les différentes approches, à laquelle viennent s'ajouter le fait que les méthodologies qui sont employées pour l'analyse ou la mesure des différents concepts ne se différencient pas toujours clairement. En effet, de nombreuses interrelations existent entre les différentes approches dès lors que l'on cherche à monétiser ou à objectiver, pour les mesurer. Par exemple, la détermination d'un seuil de pauvreté monétaire absolu, qui correspond à la vision monétaire s'appuie sur l'accès à un panier particulier des bien alimentaires retraçant la pauvreté de conditions de vie. De même, un accès difficile aux soins de santé retrace, certes, une pauvreté en termes de conditions de vie, mais aussi une pauvreté de potentialités car il influence l'espérance de vie. Il en est de même pour l'accès à l'éducation qui contribue aussi à la constitution du capital humain.

En termes de mesure statistique, on distingue entre les approches objective et subjective de la pauvreté. L'approche objective mesure le niveau de pauvreté par des instruments appropriés : seuil de pauvreté monétaire, scores des conditions de vie, indicateurs des potentialités. Quant à l'approche subjective, sont considérés comme pauvres, ceux qui se sentent pauvres dans un contexte donné. Dans tous les cas, les raisons sont diverses: insuffisance d'argent pour se procurer un produit donné (aspect monétaire), désir d'être au même niveau du voisin (aspect conditions de vie), envie de relations sociales équivalentes à celles du voisin. Au lieu de déterminer le niveau de pauvreté de façon objective par des indicateurs mesurant le niveau de vie, les conditions de vie, ou l'état des potentialités, on cherche à l'obtenir à partir des opinions des individus sur leur propre situation relative. Cette approche subjective basée sur la façon dont les ménages perçoivent leur situation, ou se perçoivent comme pauvres, vient de la nécessité de savoir comment est vécue la pauvreté et quelles interventions pourraient être mise en œuvre pour la corriger.

Notre choix méthodologique s'oriente favorablement sur l'approche objective de la pauvreté monétaire ou de niveau de vie pour les raisons suivantes (Dubois, 1998). D'un point de vue économique, cette approche méthodologique s'inscrit dans la tradition de la théorie du bien-être que l'on relie à la philosophie utilitariste. C'est le courant de pensée qui a actuellement permis le plus grand développement d'instruments d'observation, de mesure et d'analyse. De même, dans le cadre de la théorie de l'équilibre, référence est faite à la théorie micro-économique du consommateur qui maximise sa satisfaction sous contrainte budgétaire, ou du producteur-consommateur qui intègre aussi une fonction de production. Cette approche intègre de plus en plus, les dimensions sociales relatives à l'extension à l'économie de la famille et à ses interactions sociales, ainsi que des caractéristiques relevant du capital humain et du capital social.

\subsection{Les instruments d'évaluation de la pauvreté}

\section{La ligne de pauvreté}

L'approche de la pauvreté par un seuil national de la pauvreté vise à définir de manière relativement objective un but chiffré. Selon le rapport de l'INSD (2000) sur le profile de la pauvreté au Burkina Faso, "pour classer les individus selon le degré de pauvreté (des pauvres aux non pauvres), un indicateur du niveau de vie proche du revenu par tête dans un ménage a été calculé ». L'indicateur proposé est le niveau des dépenses. Il est déterminé sur la base des résultats de l'enquête comme étant égal à la somme des principales utilisations des revenus du ménage. Ce sont les dépenses en achats (acquisitions payantes) ou en 
autoconsommation des biens ou services alimentaires ou non alimentaires (santé, éducation, habitation, habillement, transport, transfert, loisirs, etc....) effectués par les membres du ménage. L'indice des dépenses ainsi défini, prend en compte toutes les utilisations possibles des revenus du ménage puisqu'il inclut en plus des acquisitions des biens et services de consommation, l'autoconsommation, l'épargne et les autres transferts. A défaut d'une estimation directe des revenus du ménage, l'indice des dépenses en donne une idée très proche. Les dépenses observées sont nominales et ont été saisies à l'échelle du ménage.

Le choix d'un seuil de pauvreté est, au moins en partie, un processus subjectif. Si on utilise un concept absolu de la pauvreté et de la consommation par tête comme mesure du bien-être, le seuil de pauvreté représenterait le niveau minimum de consommation nécessaire pour permettre à un individu d'une société donnée de subsister. La ligne de Pauvreté ou seuil absolu de pauvreté est le niveau normatif des dépenses en dessous duquel les individus sont considérés comme pauvres. Les enquêtes prioritaires I, II, et III sur les conditions de vie des ménages ont établi le seuil absolu de pauvreté respectivement à la somme de 41099 FCFA par personne et par an (INSD, 1996), de 72690 FCFA par personne et par an (INSD, 2000) et de 82672 FCFA par personne et par an (INSD, 2003), sur la base de la variation du niveau des prix et de la modification de la structure de consommation des besoins essentiels. Ces enquêtes ont été réalisées respectivement en 1994, 1998 et 2002. En termes réels, ces seuils équivalents permettent l'acquisition du même panier de biens définis qu'en 1994. Ces seuils correspondent à la couverture des besoins alimentaires (coût des dépenses nécessaires à l'obtention de 2283 calories) d'une part et des dépenses non alimentaires d'autre part (INSD, 1996).

\section{Les seuils relatifs}

37 L'approche de la pauvreté par le biais des seuils relatifs repose sur une démarche de découpage de la population totale en percentiles d'un ordre donné sur l'échelle de niveaux de vie. L'option des quintiles dans l'étude du profil de pauvreté, permet en termes monétaires, de décomposer sur l'échelle des niveaux de vie, la population en 5 fractions de $20 \%$ chacune, appelées percentiles d'ordre 5 . Le $1^{\text {er }}$ quintile représente les $20 \%$ de la population vivant dans l'extrême pauvreté, le $2^{\text {ème }}$ quintile représente les $20 \%$ de cette population vivant dans une pauvreté modérée ; le $3^{\text {ème }}$ quintile est constitué par les $20 \%$ de cette population qui ont un niveau de vie moyen; les deux derniers quintiles représentent ensembles les $40 \%$ de cette population qui vivent dans des conditions aisées de vie, c'est-à-dire les non pauvres.

\section{Les indices FGT de pauvreté}

38 Ayant défini une mesure du bien-être (Yi pour l'individu i) et déterminé un seuil de pauvreté (Z), il ne reste plus qu'à élaborer des indicateurs permettant d'appréhender les principales dimensions de la pauvreté au Burkina Faso. Ces indicateurs sont des indices spéciaux qui décrivent l'étendue, la profondeur et la sévérité. Ces indices peuvent être dérivés comme des cas particuliers de l'indice de pauvreté PX développé par Foster, Greer et Thorbecke (1984) et défini comme suit : 


$$
\mathrm{P}_{\mathrm{a}}=\frac{1}{\mathrm{n}} \sum_{\mathrm{i}=1}^{\mathrm{q}}\left(\frac{\mathrm{Z}-\mathrm{Y}_{\mathrm{i}}}{\mathrm{Z}}\right)^{\mathrm{a}}
$$
( $\mathrm{i}=\mathrm{n}$ où $\mathrm{n}$ est la population totale), $\mathrm{q}$ est le nombre d'individus considérés comme pauvres, et $\chi$ est un paramètre représentant l'importance placée sur le bien-être des plus pauvres parmi les pauvres. L'étendu de la pauvreté (la proportion de la population définie comme étant pauvre; $P o=q / n)$ peut être obtenu comme un cas spécial où $X=0$, tandis que la profondeur de la pauvreté peut être définie comme P1 et la sévérité comme étant P2. sous-groupes exclusifs et exhaustifs il est possible de calculer pour chaque sous-groupe $k$ $(k=1 . ., m)$ ayant une proportion de population égale à $\mathrm{x}_{\mathrm{k}}$.

41 Pour tout sous-groupe $k$ donné

$$
P_{i, k}=\frac{1}{n} \sum_{i=1}^{g k}\left(\frac{Z-Y_{i, k}}{Z}\right)^{\dot{a}} \Rightarrow P_{i}=\sum_{1}^{m} x^{m} P_{i, k}
$$

Par suite la contribution de chaque sous-groupe $\left(c_{k}\right)$ peut s'obtenir en écrivant :

$$
\mathrm{C}_{k}=\frac{x_{k} P_{\alpha, k}}{P_{\alpha}}
$$

43 La contribution est très utile pour identifier l'affectation différenciée de chaque sousgroupe par la pauvreté.

\section{Analyse de la relation environnement-pauvreté}

Dans cette section, nous montrons comment l'aménagement forestier au Burkina contribue à apaiser la pauvreté dans les zones forestières aménagées, notamment à travers une analyse de la structure des revenus des exploitants forestiers (4.1.) et une évaluation de la pauvreté dans ces zones (4.2.).

\section{1. Contribution des activités forestières au bien-être des exploitants forestiers}

Dans cette sous section, nous faisons une étude statistique des niveaux de vie des bûcherons et de la structure de leurs revenus. Le principal objectif est de mettre en évidence l'importance des revenus issus de l'exploitation de la ressource ligneuse mais aussi de ceux issus des ressources forestières non ligneuses regroupées dans le poste « autres revenus ». La figure $\mathrm{n}^{\circ} 1$ donne la structure des revenus des producteurs du boisénergie dans les zones forestières de production du bois-énergie.

Figure 1 : Structure des revenus des bûcherons du bois-énergie dans les sept zones forestières aménagées du Burkina en 2004. 


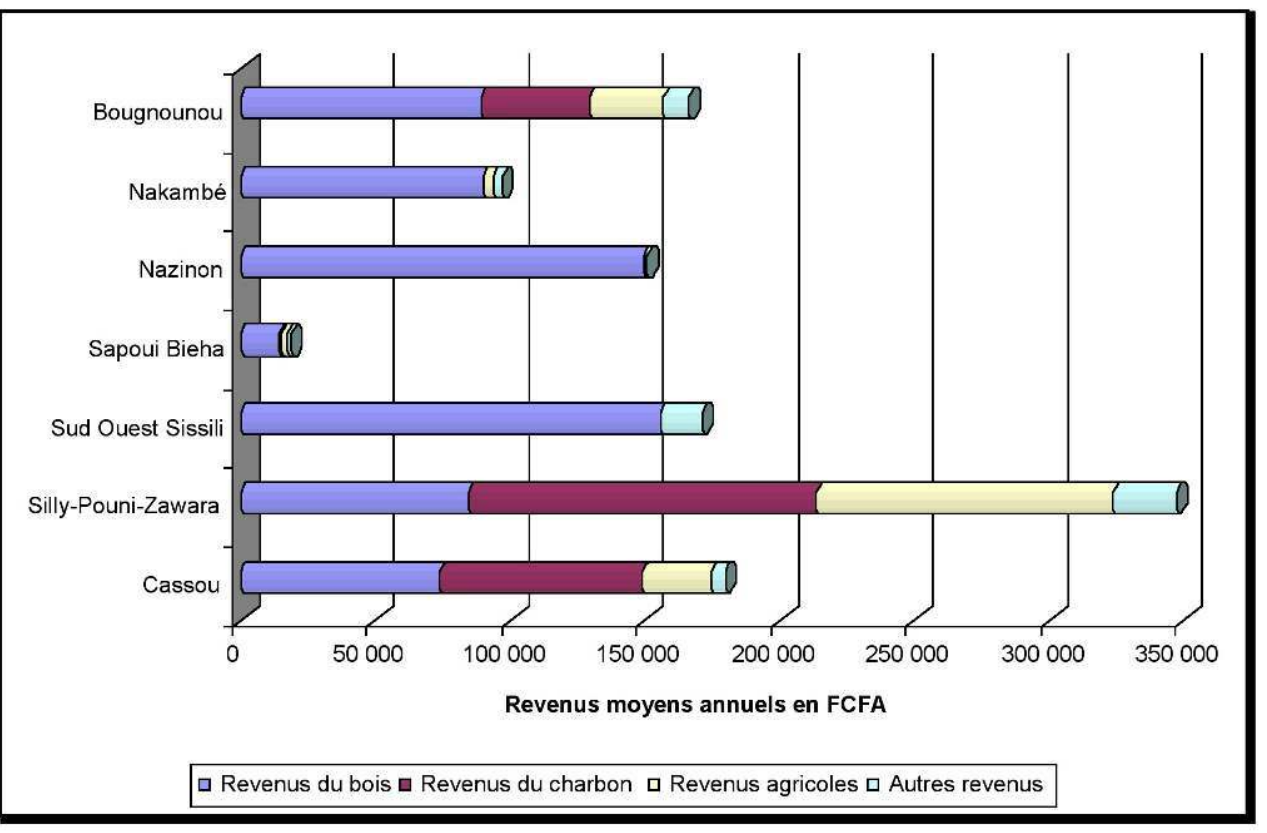
forestières approvisionnant Ouagadougou en bois de feu et en charbon de bois durant l'année 2004 qui a précédé la période d'enquête, a été de 166276 FCFA par producteur toutes activités confondues. Ce revenu est essentiellement composé par : 1) le revenu issu de la coupe du bois avec une moyenne de 89176 FCFA par bûcheron; 2) le revenu de carbonisation ou production du charbon de bois, soit en moyenne 40223 FCFA par charbonnier ; 3) le revenu agricole (vente des céréales produits par les bûcherons ou charbonniers), d'une moyenne annuelle de 27483 FCFA par producteur ; 4) les revenus issus de la cueillette (néré, karité, de l'apiculture, des autres produits forestiers non ligneux, de la production de fourrages et autres, qui contribuent en moyenne d'un montant annuel de 9614 FCFA.

lly a une forte dispersion des revenus moyens de ces producteurs entre zones forestières d'exploitation du bois-énergie, entre villages producteurs de cette énergie et entre activités. L'écart type global du revenu moyen total donne un coefficient de variation de l'ordre de 179,30 \% entre les chantiers : ce qui montre une forte disparité des revenus des producteurs du bois-énergie entre les chantiers. Cette grande disparité des revenus s'explique par les différences d'opportunités économiques observées dans la zone forestière (activités agricoles de rente, apiculture, zones potentielles de collecte de karité et de néré, production pépinière, etc...). En effet, parmi les sept zones forestières autonomes, deux zones dont le chantier Silly-Pouni-Zawara et celui du Sud Ouest Sissili font des activités de carbonisation. Les revenus moyens annuels de ces deux zones sont les plus élevés et sont respectivement évalués à 179308 FCFA et de 347573 FCFA par exploitant (voire annexes tableau $n^{\circ} 1$ ). Ces deuxzones sont suivies en termes de revenus par les chantiers de Sapouy Biéha, du Nakambé, de Bougnounou et de Kassou. Le chantier au revenu moyen annuel par exploitant le plus bas est celui de du Nazinon.

Dans l'ensemble des zones forestières enquêtées, le revenu moyen total de l'exploitant est composé de 70,47\% des revenus issus de la coupe du bois, 12,81\% des revenus issus de la carbonisation pour la fabrication du charbon de bois, 9,73\% de revenus agricoles et $6,97 \%$ de revenus issus d'autres activités forestières. Là également il y a une forte 
disparité de la structure des revenus entre les zones forestières. Selon que l'exploitant est un bûcheron ou un charbonnier sa structure des revenus est différente; mais pour la zone de Silly-Pouni-Zawara, la coupe de bois contribution en moyenne pour $57,86 \%$ dans le revenu total de l'exploitant, la carbonisation pour $29,15 \%$, l'agriculture pour $8,33 \%$ et $4,6 \%$ pour les autres activités forestières. Dans le chantier du Sud Ouest Sissili, la composition de la structure des revenus est plus ou moins équilibrée entre les postes donnant respectivement une contribution moyenne de $33,27 \%$ pour la coupe de bois dans le revenu total de l'exploitant, de $30,98 \%$ pour la carbonisation, de $\% 26,71 \%$ pour l'agriculture et de $9,03 \%$ pour les autres activités forestières (voire annexes tableau n ${ }^{\circ} 2$ ). Figure 2: Revenus des exploitants forestiers par chantiers forestiers aménagés et par type d'activité

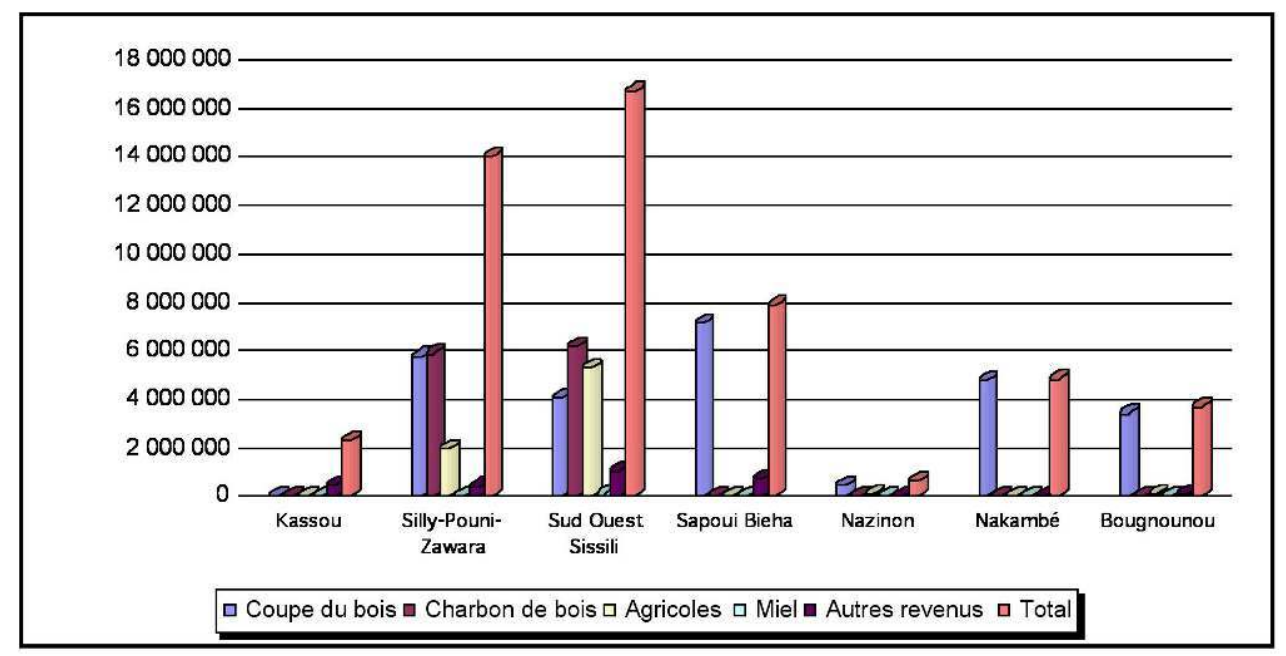

49 Ces deux zones forestières donnent de plus grandes opportunités économiques que les autres zones forestières d'exploitation du bois-énergie pour les régions suivantes: d'abord, du fait que ces zones plus éloignées de la ville de Ouagadougou, il leur était permis de produire du charbon de bois; ensuite, ce sont deux zones d'importance agricole et enfin, dans ces zones les caractéristiques végétales offrent des opportunités pour la cueillette du karité, du néré et de la production apicole. La figure $\mathrm{n}^{\circ} 2$ donne par zone forestière et par type d'activité, les revenus totaux.

Les 300 bûcherons et charbonniers des sept chantiers forestiers ont reçu des revenus totaux de 50,1 millions de FCFA dont près de 27 millions au titre de la coupe du bois, 12,1 millions au titre de la carbonisation, 8,245 millions au titre des activités agricoles, 28 millions au titre des autres activités forestières et 98000 FCFA pour la production apicole. Dans l'ensemble des zones forestières de production du bois-énergie, le revenu moyen annuel des bûcherons et charbonniers est très élevé avec quand même une très forte disparité de ces revenus entre zones forestières et villages de résidence de ceux-ci, mais entre activités constituant les principaux postes de revenus de ceux-ci.

\subsection{Analyse de la pauvreté dans les zones forestières}

51 Dans cette section, nous étudions la pauvreté dans les chantiers forestiers aménagés respectivement par les seuils relatifs et les indices FGT de pauvreté. 


\section{Analyse de la pauvreté à l'aide des percentiles}

L'option des percentiles dans l'étude du profil de pauvreté permet de décomposer sur l'échelle des niveaux de vie, la population en 10 fractions de $10 \%$ chacune. Le graphique ci-dessous donne les percentiles d'ordre dix qui analyse les degrés de pauvreté parmi les bûcherons.

Figure 3: Courbe de concentration des revenus des bûcherons. Données mai-juin 2005.

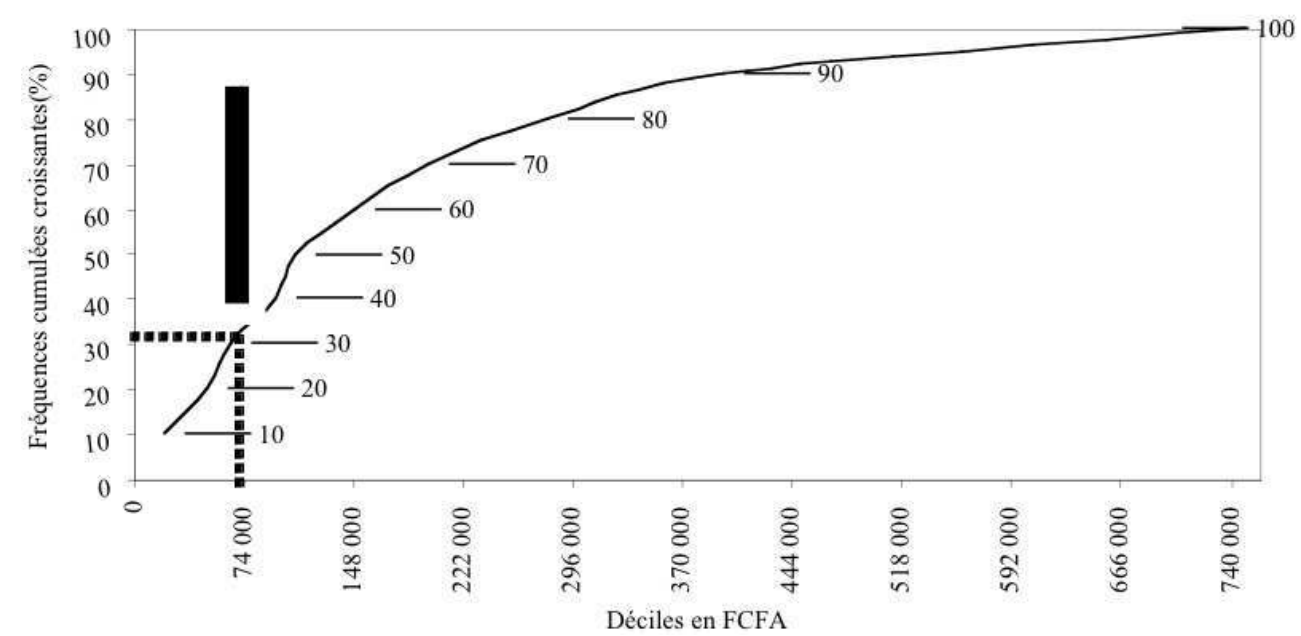

En effet, $30 \%$ des exploitants forestiers ont un revenu moyen annuel inférieur à 65300 FCFA et $40 \%$ des exploitants ont un revenu moyen annuel inférieur à 97000 FCFA. Or le seuil de pauvreté national est établi à 82672 FCFA en 2003 : ce qui veut dire qu'en utilisant la méthode d'extrapolation linéaire, on peut calculer la proportion des bûcherons en dessous de ce seuil qui se situe entre 30 \% et 40 \% de ceux-ci. La méthode de calcul est la suivante :

$$
\frac{82672-65300}{97000-65300}=\frac{X-30 \%}{40 \%-30 \%} \Rightarrow X=35,48 \%
$$

$54 \mathrm{X}$ étant la proportion des bûcherons se situant en dessous du seuil de pauvreté, alors on peut dire $35,48 \%$ des bûcherons reçoivent un revenu moyen annuel inférieur à 82 672FCFA. Le point A de coordonnées (82 672 FCFA, 35,48\%) sur le graphique de la courbe de concentration indique cette proportion. Ce résultat conforte notre troisième hypothèse puisque environ 64,52 \% des bûcherons se situent au dessus de ce seuil.

Le revenu médian est de 109750 FCFA, revenu qui divise les 300 exploitants en deux parties égales: ce qui indique que $50 \%$ des exploitants ont un revenu moyen annuel supérieur à 109750 FCFA dont les $30 \%$ reçoivent un revenu moyen annuel supérieur à 200000 FCFA et inférieur ou égal 750000 FCFA. On observe que parmi les bûcherons dont le revenu moyen annuel est au dessus du seuil de pauvreté, il y a une disparité des niveaux de vie de ceux-ci. Ces conclusions ont nécessité l'utilisation des indices de pauvreté pour une analyse plus poussée de ce phénomène. 


\section{Analyse de la pauvreté à l'aide d'un indice FGT de pauvreté}

Afin de mieux appréhender l'état de la pauvreté au sein des exploitants du bois-énergie la détermination des indices de pauvreté s'avère nécessaire. Les indices sont calculés à partir de la formule développée par Foster, Greer et Thorbecke (FGT) en 1984. Ces indices de pauvreté FGT sont largement employés dans la littérature. L'interprétation des niveaux d'indices FGT diffère selon l'importance donnée à l'inégalité entre les membres d'une population. Les principaux indices de la famille FGT sont l'indice numérique de pauvreté (l'incidence), l'écart moyen du seuil de pauvreté (la profondeur) et l'indice de sévérité de pauvreté. Nous rappelons l'équation 1 suivante :

$P_{\alpha}\left(Z, Y_{i}\right)=\frac{1}{n} \sum_{i}^{q}\left(\frac{Z-Y_{i}}{Z}\right)^{\alpha}$

Image 11 = diversion pour la pauvreté

Image12 = seuil de pauvreté (82 672 FCFA)

Image 13 = revenu de l'individu $\mathrm{i}$

Image $14=$ nombre de pauvres

Image 15 = effectif de la population

57 L'incidence (P0), la profondeur (P1) et la sévérité (P2) de la pauvreté se calculent respectivement en remplaçant respectivement dans l'équation $1 \alpha$ par 0,1 et 2 . Ainsi, on obtient respectivement ces trois indices dans les équations 4,5 et 6 ci-dessous :

$$
\begin{aligned}
& P_{0}=\frac{1}{n} \sum_{1}^{q}\left(\frac{Z-Y_{i}}{Z}\right)^{0} \Rightarrow P_{0}=\frac{q}{n} \\
& P_{1}=\frac{1}{n} \sum_{1}^{q}\left(\frac{Z-Y_{i}}{Z}\right) \\
& P_{2}=\frac{1}{n} \sum_{1}^{q}\left(\frac{Z-Y_{i}}{Z}\right)^{2}
\end{aligned}
$$

Une application numérique des équations 4,5 et 6 sur chacune des sept zones forestières

\begin{tabular}{|c|c|c|c|c|c|c|c|c|}
\hline \multirow{2}{*}{ Zone forestière } & \multirow{2}{*}{$\begin{array}{c}\text { Effectif } \\
\text { (n) }\end{array}$} & \multirow{2}{*}{$\begin{array}{l}\text { Pauvres } \\
\text { (q) }\end{array}$} & \multicolumn{2}{|c|}{$\begin{array}{l}\text { Incidence de } \\
\text { pauvreté }(\%)\end{array}$} & \multicolumn{2}{|c|}{$\begin{array}{c}\text { Profondeur de } \\
\text { pauvreté }(\%)\end{array}$} & \multicolumn{2}{|c|}{$\begin{array}{c}\text { Sévérité de } \\
\text { pauvreté }(\%)\end{array}$} \\
\hline & & & $P_{0}$ & $\mathrm{C}_{0}$ & $P_{1}$ & $\mathbf{C}_{1}$ & $\mathbf{P}_{2}$ & $\mathrm{C}_{2}$ \\
\hline Cassou & 25 & 15 & 60,00 & 13,39 & 33,10 & 15,65 & 20,36 & 14,67 \\
\hline Silly-Pouni-Zawara & 77 & 19 & 24,68 & 16,96 & 7,76 & 11,30 & 3,10 & 6,88 \\
\hline Sud Ouest sissili & 48 & 3 & 6,25 & 2,68 & 1,59 & 1,44 & 0,50 & 0,69 \\
\hline Sapoui Bieha & 46 & 7 & 15,22 & 6,25 & 6,08 & 5,29 & 3,50 & 4,65 \\
\hline Nazinon & 34 & 34 & 100,00 & 30,36 & 77,05 & 49,54 & 65,08 & 63,77 \\
\hline Nakambé & 32 & 8 & 25,00 & 7,14 & 6,78 & 4,10 & 2,17 & 2,00 \\
\hline Bougnounou & 38 & 26 & 68,42 & 23,21 & 17,65 & 12,68 & 6,70 & 7,34 \\
\hline Ensemble & 300 & 112 & 37,33 & 37,33 & 17,63 & 17,63 & 11,57 & 11,57 \\
\hline
\end{tabular}
étudiées permet d'obtenir le tableau $\mathrm{n}^{\circ} 1$ ci-dessous.

Tableau 3 : Les indices de pauvreté FGT dans les sept zones forestières aménagées du Burkina

Source : Analyse des données d'enquêtes bûcherons (mai-juin 2005). 


\section{L'incidence de pauvreté (IP)} forestières en ses colonnes 2 et 3 . L'incidence de la pauvreté dans les 7 zones forestières et sur les 300 producteurs du bois-énergie enquêtés est de $37,33 \%$. Cela signifie que $37,33 \%$ de la population est pauvre : ce taux est légèrement supérieur à celui de 35,48 \% approximé par la méthode des percentiles. Ce taux reste inférieur à l'incidence de la pauvreté au niveau rural qui est de 52,3\% (INSD ; 2003). L'indice de pauvreté s'observe avec une forte disparité entre les zones forestières, et cela est le reflet de celle observée au niveau des revenus moyens annuels des bûcherons. Là également, l'incidence de pauvreté le plus bas s'observe dans les zones forestières les plus nanties avec respectivement 6,25\% dans le chantier du Sud Ouest Sissili, de 15,22 \% à Sapoui-Biéha et $24,68 \%$ au chantier de Silly-Pouni-Zawara où les revenus moyens annuels des exploitants sont les plus élevés. Les zones forestières où l'incidence de pauvreté est la plus importante sont celles de Cassou avec un seuil de $60 \%$ et celle du Nazinon avec une incidence de $100 \%$, tous les bûcherons enquêtés dans ce dernier chantier forestier se situant en dessous de la ligne de pauvreté calculée de 2003. Cette forte disparité est également observée lorsqu'on calcule les incidences au niveau des villages enquêtés. La figure $\mathrm{n}^{\circ} 4$ donne pour les différents villages riverains, l'incidence de la pauvreté et sa contribution (voire également tableau annexe $n^{\circ} 4$ en ses colonnes $n^{\circ} 6$ et 7 ).

Figure 4 : Incidence de la pauvreté dans les différents villages riverains des sept forêts aménagées

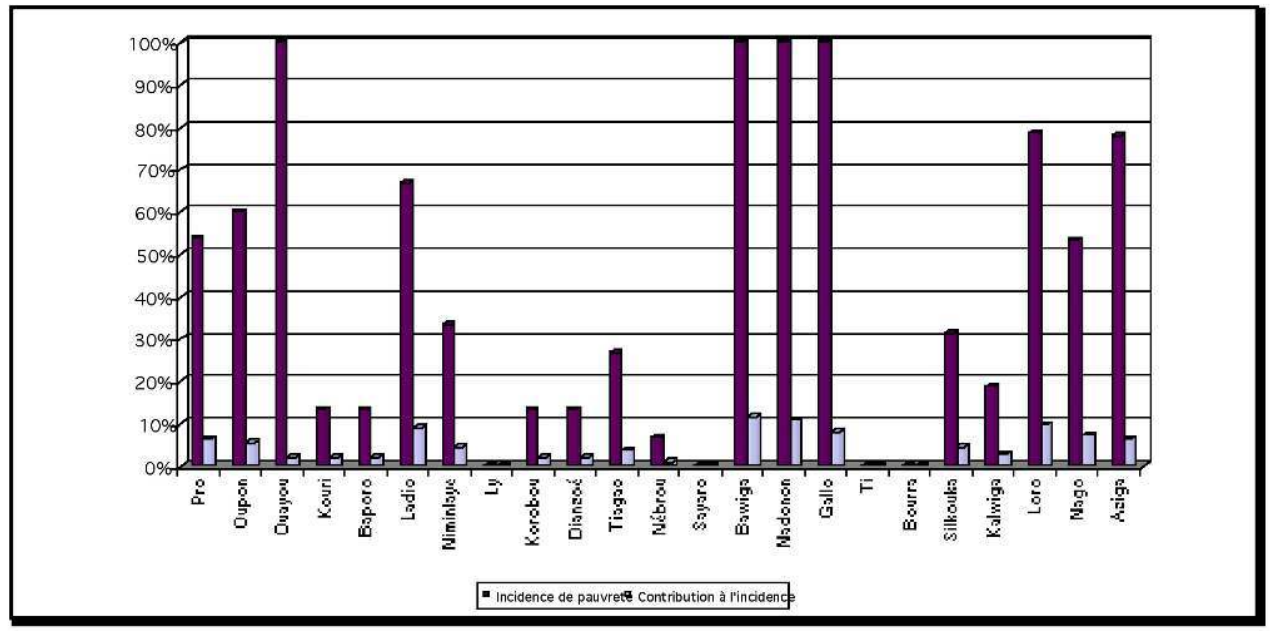
respective de $100 \%$, ce qui montre les revenus moyens annuels de ces villages par exploitant sont situés au-dessous du seuil absolu de pauvreté.

La contribution de ces villages les moins nantis au taux de pauvreté d'ensemble est respectivement de 1,79\% pour Ouayou, 11,61 \% pour de Nadanon, de 10,71 \% pour Bawiga et de de $8,04 \%$ pour Gallo : la faible contribution du village d'Ouayou étant dû au nombre très réduit de 2 bûcherons enquêtés dans ce village. Des villages comme Ly, Sayaro, Ti et Bourra ont enregistré paradoxalement des incidences de pauvreté $0 \%$, autrement dit tous les exploitants de ces villages ont un revenu moyen annuel supérieur au seuil de pauvreté. Ly, Ti et Boura sont des villages de la zone forestière du Sud Ouest Sissili qui est l'une des zones les plus nanties. Nébrou et Sayaro sont de Spaouy Biéha. 


\section{La profondeur de la pauvreté}

62 les zones étudiées (voire statistiques de ces indices de pauvreté dans les colonne 4 et 5 du
tableau annexé $n^{\circ} 5$ ).

Figure 5 : Profondeur de la pauvreté dans les sept zones forestières enquêtées

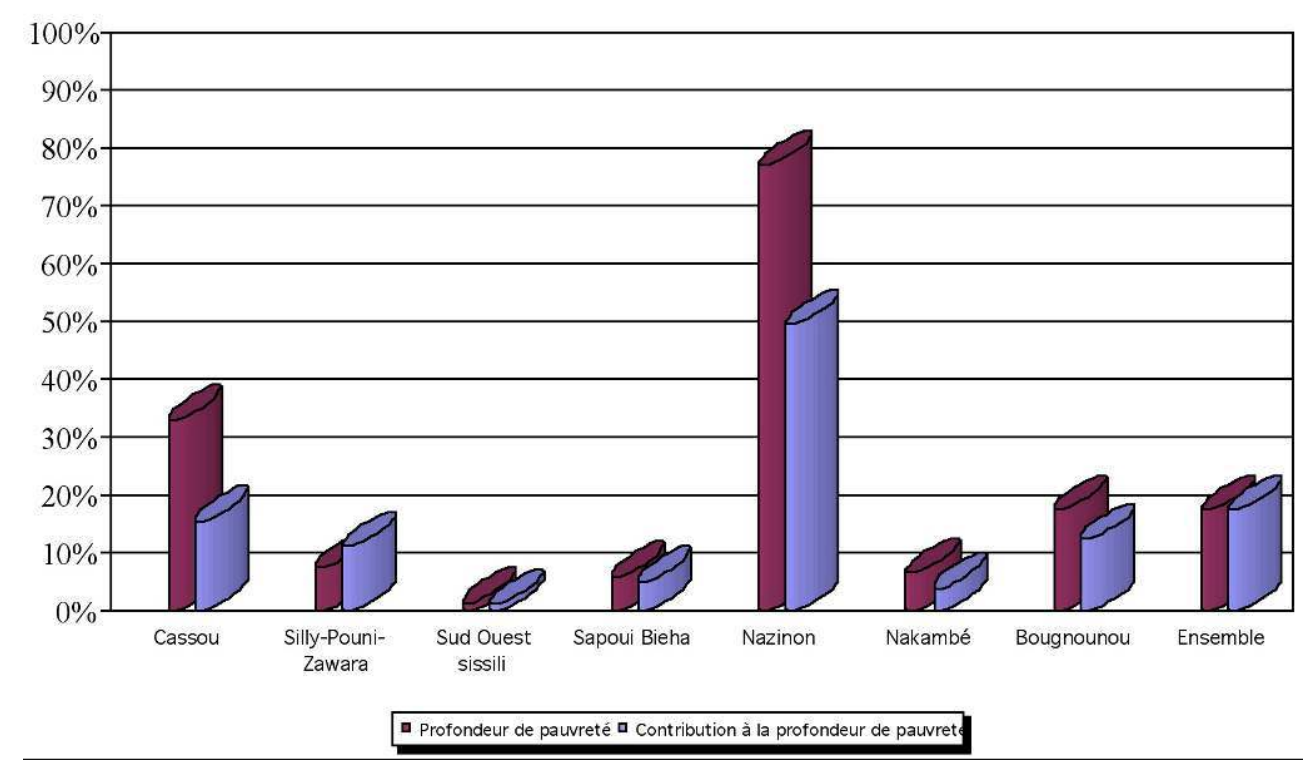

Ravallion (1996) donne une interprétation très concrète de l'écart moyen du seuil de pauvreté, le considérant comme étant le coût minimal pour éliminer la pauvreté en utilisant des transferts ciblés qui sont constitués simplement de la somme de tous les écarts de pauvreté. La profondeur de la pauvreté ou « poverty gap » dans l'ensemble des 7 zones forestières et sur les 300 producteurs du bois-énergie enquêtés est de 15,32 \%. Ce taux est reste inférieur à celui de la profondeur de la pauvreté au niveau rural national qui est de l'ordre de 17,9\% (INSD ; 2003). La profondeur de la pauvreté indique par rapport au seuil absolu de la pauvreté, le pourcentage de variation du revenu ou de la dépense annuels des personnes pauvres. Ainsi, une profondeur de la pauvreté de 15,32\% indique que la moyenne de la variation en $\%$ du revenu annuel des pauvres par rapport au seuil de pauvreté est de $15,32 \%$.

Il est également observé à ce niveau une grande disparité des taux à ce niveau. Les zones forestières où la pauvreté est la plus profonde sont celle du Sud Ouest Sissili, de SylliPouni-Zawara, du Nakambé et de Sapouy Biéha. C'est dans la zone forestière du Nazinon qu'on observe une pauvreté dont la profondeur est la plus importante de toutes les zones forestières. Cette même disparité de la profondeur de la pauvreté est observée au niveau des villages des zones forestières exploitées. Dans certains villages comme Ly, Ti et Bourra, elle est pratiquement nulle, ce qui confirme l'aisance des exploitants vivant dans ces localités qui en réalités présentent de meilleures opportunités économiques que les autres. 


\section{La sévérité de la pauvreté}

\section{forestiers aménagés (voire statistiques de ces indices de pauvreté dans les colonne 6 et 7} du tableau annexé $\left.\mathrm{n}^{\circ} 5\right)$.

Figure 6 : Indice FGT de sévérité de pauvreté et sa contribution à la sévérité de pauvreté dans les 7 zones forestières

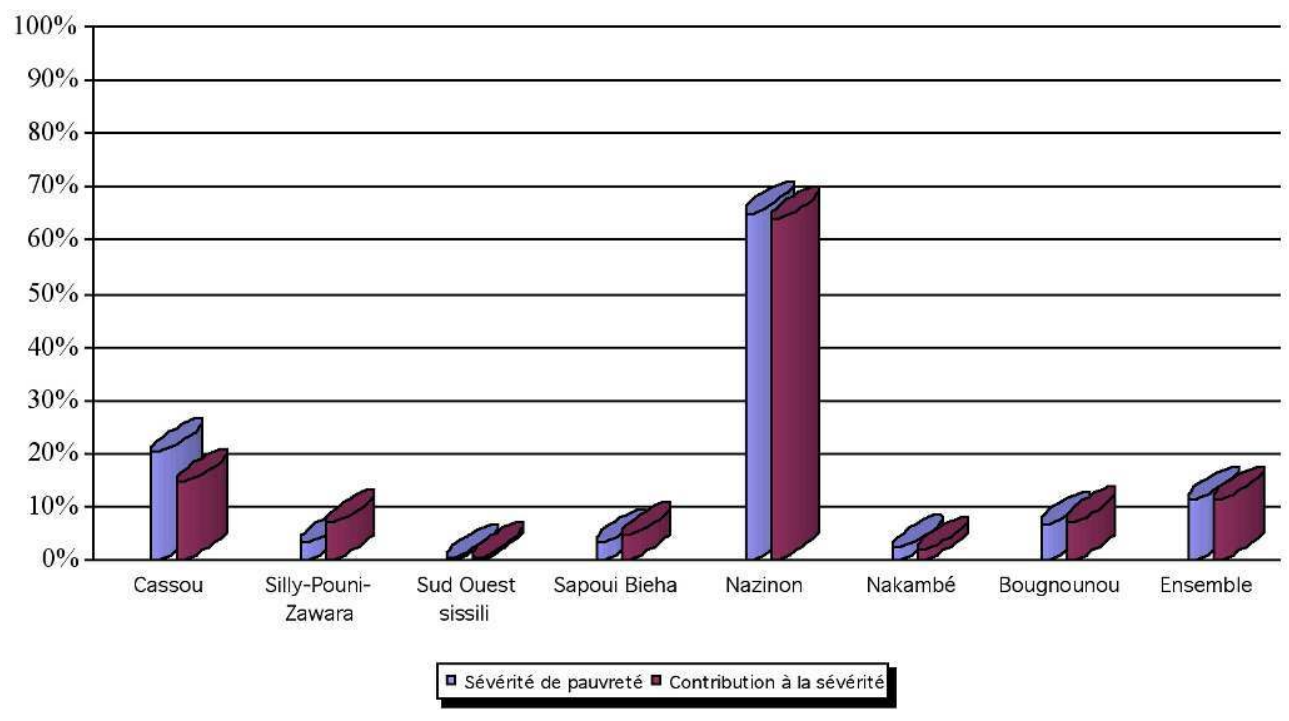

La sévérité de la pauvreté est de 10,12\% dans l'ensemble des 7 zones forestières. Cet indicateur est très élevé comparé au seuil national de sévérité de la pauvreté dont le niveau rural est de $6,8 \%$. (INSD, 2003). Cet indice indique en moyenne la variabilité des revenus des personnes pauvres par rapport au seuil de pauvreté; en d'autres termes, il montre que parmi les personnes pauvres il y a une diversité de la pauvreté. Une sévérité de $10,12 \%$ indique que parmi les personnes pauvres, le niveau de la pauvreté des ces individus varie en moyenne de 10,12 \% d'un individu à l'autre.

On observe également entre les différentes zones forestières une forte disparité de cet indicateur. Par exemples dans les chantiers forestiers du Sud Ouest Sissili, de Silly Pouni Zawara, du Nakambé, et de Sapouy Biéha, On observe une faible sévérité de la pauvreté qui se situe entre $0,27 \%$ et $2 \%$ qui indiquent entre les personnes pauvres une certaine homogénéité de la pauvreté, en ce sens que la moyenne du \% de la somme du carré des écarts entre le seuil de pauvreté et le revenu des individus pauvres sur le seuil de pauvreté est très faible. D'un individu pauvre à un autre, ce \% est de l'ordre de $0,27 \%$ à $2 \%$. La sévérité de la pauvreté est la plus forte dans le chantier du Nazinon, avec un seuil de $61,94 \%$, ce qui indique une forte disparité des niveaux de pauvreté parmi les personnes pauvres de cette zone forestière, donc une grande hétérogénéité de la pauvreté parmi les exploitants forestiers de cette zone.

\section{Une expression de la pauvreté dans les zones forestières selon le type d'exploitant}

Les indices $\mathrm{P}_{\chi}$ de pauvreté FGT sont décomposables. Lorsque la population totale peut être répartie en $m$ sous-groupes exclusifs et exhaustifs, il est possible de calculer pour chaque sous-groupe $k(k=1 . ., m)$ ayant une proportion de population égale à $\mathrm{x}_{\mathrm{k} \text {. }}$ Les équations 2 et 
3 donnent la formule de calcul des indices décomposés et celle de la contribution de chaque sous-groupe. Pour tout sous-groupe k donné,

$$
\mathrm{P}_{\mathrm{a}, \mathrm{k}}=\frac{1}{\mathrm{n}} \sum_{\mathrm{i}=1}^{\mathrm{q} \mathrm{k}}\left(\frac{\mathrm{Z}-\mathrm{Y}_{\mathrm{i}, \mathrm{k}}}{\mathrm{Z}}\right)^{\mathrm{a}} \Rightarrow \mathrm{P}_{\mathrm{a}}=\sum_{\mathrm{l}}^{\mathrm{m}} \mathrm{xkP}_{\mathrm{a}, \mathrm{k}}
$$

$$
\mathrm{C}_{k}=\frac{x_{k} P_{\alpha, k}}{P_{\alpha}}
$$
groupe par la pauvreté. L'utilisation des indices décomposés dans ce travail va permettre d'appréhender la pauvreté au sein des exploitants selon le type de bois-énergie produit (bois de feu et charbon de bois). Il faut noter que la carbonisation est une activité qui n'est pas permise dans toutes les zones forestières aménagées, exceptées celles qui sont situées à plus de $100 \mathrm{~km}$ des principaux centre urbains. Cela est dû aux très faibles rendements des techniques de carbonisation, ne rendant le charbon de bois socialement rentable qu'à des distances de coupe minimum de $100 \mathrm{~km}$ de ces centres de grande consommation. Une application numérique des équations permet d'obtenir les indices FGT décomposés et leurs contributions respectives dans le tableau 4 ci-dessous.

En décomposant les indices de pauvreté, on observe que l'incidence de pauvreté FGT est la plus forte chez les bûcherons exploitant uniquement le bois avec une incidence de l'ordre $41 \%$ contre une incidence de $8,82 \%$ chez les charbonniers qui procèdent à la carbonisation du bois après sa coupe. Chez les bûcherons, la contribution au taux de pauvreté est de $97 \%$ contre seulement 2,68\% chez les producteurs de charbons de bois. L'étendue de la pauvreté est également la plus accrue chez les bûcherons avec une profondeur de pauvreté de $19,48 \%$ et une contribution de l'ordre $98 \%$ par rapport aux charbonniers qui présentent une faible étendue de pauvreté de l'ordre de 3,13\% et une contribution de $2 \%$.

\begin{tabular}{|c|c|c|c|c|}
\hline \multirow{2}{*}{\multicolumn{2}{|c|}{ Indicateurs }} & \multicolumn{2}{|c|}{ Types d'exploitant forestiers } & \multirow{2}{*}{ Total } \\
\hline & & Bûcherons & Charbonniers & \\
\hline \multirow{4}{*}{ 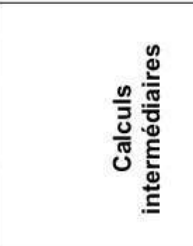 } & Effectif $(n)$ & 266 & 34 & 300 \\
\hline & Pauvres (q) & 109 & 3 & 112 \\
\hline & $A=\sum_{i}^{q}\left(\frac{Z-Y_{i}}{Z}\right)^{1}$ & 51,82 & 1,06 & 52,88 \\
\hline & $B=\sum_{i}^{q}\left(\frac{Z-Y_{i}}{Z}\right)^{2}$ & 34,31 & 0,39 & 34,70 \\
\hline \multirow{2}{*}{ Incidence (\%) } & Incidence de la pauvreté $\left(P_{0}\right)$ & 40,98 & 8,82 & \multirow{2}{*}{37,33} \\
\hline & Contribution $\left(\mathbf{C}_{0}\right)$ & 97,32 & 2,68 & \\
\hline \multirow{2}{*}{ Profondeur (\%) } & Profondeur de la pauvreté $\left(\mathbf{P}_{1}\right)$ & 19,48 & 3,13 & \multirow{2}{*}{17,63} \\
\hline & Contribution $\left(\mathbf{C}_{1}\right)$ & 97,99 & 2,01 & \\
\hline \multirow{2}{*}{ Sévérité (\%) } & Sévérité de la pauvreté $\left(P_{2}\right)$ & 12,90 & 1,14 & \multirow{2}{*}{11,57} \\
\hline & Contribution $\left(\mathrm{C}_{2}\right)$ & 98,88 & 1,12 & \\
\hline
\end{tabular}

Tableau 4 : Indices FGT décomposés de pauvreté selon le type d'exploitant forestier

72 Les indices décomposés révèlent une forte contribution à la sévérité de la pauvreté chez les bûcherons (environ $99 \%$ ) par rapport aux charbonniers (1,12\%). La sévérité de la 
pauvreté chez les bûcherons reste plus élevée que la moyenne rurale nationale de l'ordre de $9,6 \%$, tandis chez les charbonniers, cet indice reste très faible $(1,14 \%)$.

\section{Eléments de conclusion et de discussion.}

Après plus de deux décennies d'expérience d'aménagement des forêts naturelles basée sur une approche participative des populations riveraines, et, visant l'approvisionnement des populations urbaines en bois-énergie et la sauvegarde de l'environnement, les questions sur la durabilité de ces forêts et la contribution de l'aménagement à la réduction de la pauvreté relèvent d'un intérêt public avéré pour le Burkina Faso. La concession de la gestion des zones forestières aménagées aux groupements villageois de gestion forestière et la poursuite encore aujourd'hui de l'aménagement de nouvelles forêts classées (667 600 hectares sont aménagées et 202400 hectares sont en aménagement), justifient la pertinence et la durabilité des méthodes d'aménagement adoptées. Cependant, malgré les considérations et/ou l'intégration de l'exploitation forestière dans les Objectifs du Millénaires pour le Développement (OMD) et la mise en œuvre du Cadre Stratégique de Lutte contre la pauvreté (CSLP) au Burkina, aucune étude utilisant des outils d'analyse de la pauvreté avant celle-ci ne s'était penché sur l'analyse de la contribution spécifique de l'exploitation forestière sur la réduction de la pauvreté parmi les populations riveraines de ces forêts aménagées au Burkina Faso. Les conclusions de cette étude sont nombreuses.

D'une part, l'aménagement des forêts naturelles dans ce pays a contribué à générer de nouvelles sources de revenus pour les populations riveraines de ces forêts. En effets, le poste "autres revenus" a donné une moyenne annuelle par bûcheron de 9614 FCFA. Cette moyenne est très variable entre chantiers, entre villages d'un même chantier et entre différents types d'exploitants (bûcherons ou charbonniers) : elle atteint 23719 FCFA dans le chantier du Sud Ouest Sissili. Ce poste regroupe les revenus issus de la cueillette de fruits sauvages (néré, karité), des activités de l'apiculture organisées dans le chantier, de la récolte des graines d'arbres, de la production pépinière, de la production de fourrages, de la construction des pare-feux. La majeure partie de ces activités résulte des initiatives et de l'organisation de la direction technique des chantiers forestiers aménagés. Cette activité de cueillette et de production (apiculture) dont la commercialisation concerne en majorité les femmes, procure d'importants revenus à cette catégorie vulnérable de la population de nos pays. Bien que les espèces végétales concernées (Butyropermum paradoxum, Detarium microcarpum, Bombax costatum, Balanites aegyptiaca et Adansonia digitata, etc..) soient déjà protégées par les textes et lois en vigueur dans le pays, elles doivent faire l'objet de politiques spécifiques afin de pérenniser l'activité. En plus il faut noter l'importance du Butyropermum paradoxum dont l'amende et le beurre sont une importante source d'entrée de devises pour le pays.

D'autre part, l'examen de la structure des revenus des bûcherons révèle une prédominance des revenus de sources forestières sur les autres sources de revenus de l'exploitant forestier. Le revenu moyen annuel total de celui-ci est composé de 70,47\% des revenus issus de la coupe du bois, $12,81 \%$ des revenus issus de la carbonisation pour la fabrication du charbon de bois, $9,73 \%$ de revenus agricoles et $6,97 \%$ de revenus issus d'autres activités forestières, soit au total une contribution de l'exploitation du boisénergie pour $83,28 \%$ au revenu moyen annuel du producteur de bois-énergie. La contribution de l'ensemble des revenus forestiers à la structure des revenus atteint 
$90,25 \%$ du revenu moyen total de ces exploitants forestiers. En outre, même les populations riveraines n'intervenant pas directement dans l'exploitation forestière bénéficient de cette activité à travers le fond de roulement ou fonds d'investissement villages prélevé sur chaque stère de bois coupé et commercialisé dans les zones forestières aménagées. Le fonds d'investissement ou fonds de roulement, ou encore caisse villageoise, d'un montant de 200FCFA par stère coupé et vendu, a pour finalité l'amélioration du bien-être collectif du village et vise notamment à créer et/ou à entretenir des infrastructures sociales tels que les écoles, les forages et les dispensaires etc.... Les seuls éléments ci-dessus relevés, permettent d'affirmer que l'exploitation forestière contribue dans les zones forestières aménagées à améliorer les conditions de vie des populations riveraines de ces forêts.

Enfin, le calcul et l'utilisation des indicateurs FGT de pauvreté sur 300 exploitants forestiers d'une vingtaine de villages riverains des forêts aménagées permettent de saisir avec pertinence l'incidence, la profondeur et la sévérité de la pauvreté dans ces zones aménagées. Les indices FGT de pauvreté ainsi calculés et leur décomposition permettent d'observer une disparité de l'incidence, de la profondeur et de la sévérité de pauvreté parmi les exploitants forestiers d'un même village, parmi ceux de villages différents, parmi ceux de chantiers forestiers différents, et même parmi différents types d'exploitants (bois de feu et charbon de bois). Cette disparité s'explique d'une part par les différences de potentialités forestières et agroclimatiques qu'offrent les différentes zones forestières aménagées, et d'autre part, par les initiatives personnelles prises par les directeurs techniques des chantiers pour développer des projets de développement parmi ces exploitants (apiculture, embouche bovine, maraîchage etc....). Le taux de pauvreté dans les 7 zones forestières est de 37,33\%, taux qui reste inférieur à l'incidence de la pauvreté au niveau rural qui est de 52,3\% (INSD, 2003). L'indice de pauvreté s'observe avec une forte disparité entre les zones forestières, et cela est le reflet de celle observée au niveau des revenus moyens annuels des bûcherons.

77 La profondeur de la pauvreté dans l'ensemble des 7 zones forestières est de 15,32 \%, taux inférieur à celui de la profondeur de la pauvreté au niveau rural national qui est de l'ordre de 17,9\% (INSD, 2003). Dans les chantiers forestiers du Sud Ouest Sissili, de Silly Pouni Zawara, du Nakambé, et de Sapouy Biéha, on observe une faible sévérité de la pauvreté qui se situe entre $0,27 \%$ et $2 \%$ qui indiquent entre les personnes pauvres une certaine homogénéité de la pauvreté. Cependant, la sévérité de la pauvreté est la plus forte dans le chantier du Nazinon, avec un seuil de 61,94\% indiquant une forte disparité des niveaux de pauvreté parmi les personnes pauvres de cette zone forestière, donc une grande hétérogénéité de la pauvreté parmi les exploitants forestiers de cette zone.

Les indices décomposés de pauvreté selon le type d'exploitant montrent que l'incidence de pauvreté FGT est la plus forte chez les bûcherons exploitant uniquement le bois avec une incidence de l'ordre $41 \%$ contre une incidence de $8,82 \%$ chez les charbonniers qui procèdent à la carbonisation du bois après sa coupe. L'étendue de la pauvreté est également la plus accrue chez les bûcherons avec une profondeur de pauvreté de 19,48 \% et une contribution de l'ordre $98 \%$ par rapport aux charbonniers qui présentent une faible étendue de pauvreté de l'ordre de 3,13\% et une contribution de $2 \%$. Les indices décomposés révèlent une forte contribution à la sévérité de la pauvreté chez les bûcherons (environ $99 \%$ ) par rapport aux charbonniers (1,12\%). La sévérité de la pauvreté chez les bûcherons reste plus élevée que la moyenne rurale nationale de l'ordre de $9,6 \%$, tandis chez les charbonniers, cet indice reste très faible $(1,14 \%)$. 

bois-énergie de la ville de Ouagadougou, d'une quantité estimée à 1,38 millions de stères de bois en 2000 (Ouedraogo, 2006 ; Delnooz, 1999). Les $83 \%$ à $85 \%$ de cette demande étant couverte par une offre hors zone forestière aménagée, qualifiée d'offre non contrôlée ou de production frauduleuse (Ouédraogo, 2002 ; 2006) concerne le bois exploité à proximité des centres urbains et qui échappe aux écotaxes et à la réglementation qui sont les principaux instruments de la politique forestière au Burkina Faso. De ce fait, l'exploitation des forêts dans ces zones non aménagées, n'internalisant pas le coût de remplacement de la ressource forestière comme dans les zones forestière aménagée (Fonds d'Aménagement Forestier et Taxe Forestière par exemple), autorise une surestimation des bénéfices nets, laissant entrevoir une Tragédie des Communs comme l'indique Hardin (1968). Une étude complémentaire sur l'impact de l'exploitation du boisénergie dans ces zones forestières non aménagées sur les exploitants pourrait être réalisée.

Globalement, si les exploitants du bois-énergie dans les zones forestières aménagées sont moins pauvres que les populations rurales au Burkina, on observe parmi eux une plus grande étendue et sévérité de la pauvreté par rapport à celles des moyennes rurales du pays. Parmi ces exploitants producteurs de charbon de bois sont les plus riches et représentent une catégorie d'exploitants où l'étendue et la sévérité de la pauvreté sont très faibles. Compte tenu de la contribution de ces zones forestières à l'amélioration des conditions de vie des populations rurales et de celle de la gestion participative à la durabilité de ces zones forestières, des efforts supplémentaires de la part des autorités du Burkina Faso, devraient permettre de conforter ces acquis. En particulier, en s'appuyant sur le dispositif des chantiers forestiers autonomisés, le contenu des politiques de lutte contre la pauvreté pourrait prendre appui sur des projets de développement initiés par les GGF sous la direction des directeurs techniques de ces chantiers forestiers.

La portée de cette étude est cependant limitée dans la mesure où elle ne prend pas en compte l'ensemble des économies externes possibles engendrées par les activités d'aménagement sur les populations riveraines et leurs activités productives. On pourrait formuler dans des études postérieures des hypothèses sur les possibilités que ces activités d'aménagement peuvent significativement entraîner sur le développement des systèmes productifs agricoles dans ces zones forestières aménagées : la pratique de l'agroforesterie, la mécanisation agricole pouvant être propulsées par les revenus forestiers, tout comme la hausse des superficies emblavées et des rendements agricoles ou encore l'accroissement du volume d'emplois agricoles.

\section{BIBLIOGRAPHIE}

Atchoumgaï B., 2003. L'impact des marchés ruraux de bois de feu dans le village de Korokoro (Cercle de Dioila). Thèse MSc, Institut polytechnique rural de formation et de recherche appliquée (Ipr/Ifra), atibougou, Mali.

Barde Jean-Philippe, 1992, Economie et politique de l'environnement, PUF, Paris. 
Becker G., 1964, Human capital. A theoretical and empirical analysis with special reference to education, Columbia University Press, $187 \mathrm{p}$.

Becker G., 1974. “A Theory of Social Interaction”, Journal of Political Economy $82 \mathrm{n}^{\circ}$ 6, pp. 1063-1091.

Boutefeu, B., 2005. «L'aménagement des forêts en France : la recherche d'une gestion durable à travers l'histoire ». La revue en sciences de l'environnement, Vol $6 \mathrm{n}^{\circ} 2$.

Collins, P. H., 1992. Dictionary of Ecology and the Environment. Universal Book Stall, New Delhi, Inde, $117 \mathrm{p}$.

Delnooz, P. 2003. « Aménagement forestier et gestion communautaire des ressources naturelles : participation ou négociation? » Fondation Universitaire Luxembourgeoise, Arlon. Communication au séminaire international de l'université de Ouagadougou «Les organisations d'économie sociale dans la lutte pour la réduction de la pauvreté »

Delnooz P., 1999. « Gestion des Ressources Forestières : la Communauté, l'Etat et le Marché. Etude de Projets au Burkina Faso. » Fondation Universitaires Luxembourgeoise ; Thèse de Doctorat en Sciences de l'Environnement; Tomes $1 \& 2,545$ pages.

Dubois, J-L., 1998. « Différentes approches de la pauvreté ». Contribution à la Journées des Economistes IRD, « La Pauvreté », Paris.

Dubourdieu, J., 1997. Manuel d'aménagement forestier : gestion durable et intégrée des écosystèmes forestiers. Paris, Office National des Forêts, Editions Lavoisier, $244 \mathrm{p}$.

FAO., 1996. « Le Burkina Faso : Document de Politique Forestière Nationale », Programme de coopération technique, Rome.

Fisher, R., J., 1995. Collaborative Management of Forest for Conservation and Development.

Foley G., Floor W., Madon G., Lawali E. M., Montagne P., Touanao K., 1997. The Niger household energy project: promoting rural fuelwood markets and village management of natural woodlands. ESMAP/ World Bank Technical Paper. Energy series. No. 362. Washington DC: World Bank.

Foley G., Kerkhof P., Madougou D., 2002. "A review of rural firewood markets strategy in West Africa”. African Region Work Paper Series. No. 35. Washington DC: World Bank.

Foster J., Greer J., Thorbecke E., 1984, "A Class of Decomposable Poverty Measures", Econometrica, vol. $52 \mathrm{n}^{\circ} 1$, pp. 761-765.

Gray, B., 1998. Collaborating: Finding Common Ground for Multiparty Problems. Jossey-Bass, San Francisco, Etats Unis.

Grootaert C., 1997. "Social Capital: The Missing Link?" in Expanding the Measure of Wealth: Indicators of Environmentally Sustainable Development, World Bank, Washington, pp. 77-93.

Hautdidier B., Boutinot L., Gautier D., 2004. « La mise en place des marchés ruraux de bois au Mali : un évènement social et territorial. » L'Espace géographique, 4: 289-305.

Hautdidier B., Gautier D., 2005. "What local benefits does the implementation of rural wood markets in Mali generate?" In: African Forests Between Nature and Livelihood Resources: Interdisciplinary Studies in Conservation and Forest Management. Ros-thonen M. A. F., Dietz A. J. (éd.). The Edwin Mellen Press, p. 191-220.

Herpin N. et Verger D. (eds.), 1997, « Mesurer la pauvreté aujourd'hui », Economie et Statistique no308-309-310, INSEE, Paris, $272 \mathrm{p}$. 
INSD, 1996. Résultats des enquêtes prioritaires sur les conditions de vie des ménages de 1994.

Ouagadouogou, Burkina Faso

INSD, 2000. Résultats des enquêtes prioritaires sur les conditions de vie des ménages de 1998.

Ouagadouogou, Burkina Faso

INSD, 2003. La pauvreté en 2003. Ouagadouogou, Burkina Faso

KABORE C., 2004. Bilan des résultats de l'aménagement forestier au Burkina Faso. Ouagadougou, Burkina Faso, Dafor/Mee.

Ministère de l'Environnement et de l'Eau (MET), 2002. Rapport sur l'état del'Environnement au Burkina Faso.

Lucas R.E., 1988. "On the Mechanics of Economic Development", Journal of Monetary Economics $\mathrm{n}$

- 22 , pp. 3-42.

Ouedraogo B., 2002. Éléments économiques pour la gestion de l'offre et de la demande du bois-énergie dans la région de Ouagadougou. Thèse de doctorat unique, université de Ouagadougou/ université Montesquieu- Bordeaux IV, $380 \mathrm{p}$.

Ouedraogo B., 2006. «Étude de la demande de bois-énergie à Ouagadougou : une esquisse d'évaluation de l'impact physique et des échecs des politiques de prix. » Développement Durable et Territoires, Varia. Disponible en ligne le 20 mars 2006 (http://developpementdurable. revues.org/ sommaire61. html ?format =varia).

Ouédraogo, B., 2007. « Filière bois d'énergie burkinabé : structuration des prix et analyse de la répartition des bénéfices ", Bois et Forêts des Tropiques, N² 294 (4), pp. 75-88

Peyre S., 1999. « Une situation nouvelle pour la suberaie catalane. AssociationForêt Méditerranéenne , La feuille et l'aiguille, $n^{\circ} 31$.

Ravallion M. 1996. Comparaisons de la pauvreté, concepts et méthodes. LSMS document de travail N122. Banque Mondiale, Washington, D.C.

Ribot J.C., 1995. Le contrôle des forêts locales au Mali : analyse des politiques participatives du point de vue institutionnel. Harvard: Harvard University, RPTES, Centre for Development Studies.

Ribot J.C., 1998. “Theorizing access: Forest profits along Senegal's charcoal commodity chain.” Development and Change, 29, pp. 307-341.

Ribot J.C., 1999. "Decentralisation, participation and accountability in sahelian forestry: Legal instruments of political-administration control." Africa, 69 (1),pp. 23-65.

Ribot J.C., 2001. Sciences, use rights and exclusion: a history of forestry in Francophone West Africa. IIED Drylands Issues. No. 104. London:IIED, 2001.

Rowntree B.S., 1901, Poverty: a study of town life.

Sawadogo, L., 2006. Adapter les approches de l'aménagement durable des forêts sèches aux aptitudes sociales, économiques et technologiques en Afrique. Le cas du Burkina Faso. CIFOR/ASDI. Indonésie

Sen A. K., 1976, "Poverty: An Ordinal Approach to Measurement", Econometrica vol. 44, pp. 219-231.

Sen A.K., 1993, "Capability and Well-Being", in The Quality of Life, Clarendon Press, Oxford, pp. 30-66.

Thiam A. T., 1993, « Analyse du secteur bois-énergie au Burkina Faso », Ouagadougou : FAO. 
Valtriani P., 1993, “Un concept de pauvreté disjonctif”, Economie appliquée tome XLVI n 4/1993, PUG, Grenoble, pp. 151-154.

\section{ANNEXES}

Tableau 5 Structure des revenus des producteurs du bois-énergie pour 2004

\begin{tabular}{|l|c|c|c|c|c|c|}
\hline Chantiers & Type de producteur & Revenus du bois & $\begin{array}{c}\text { Revenus du } \\
\text { charbon }\end{array}$ & $\begin{array}{l}\text { Revenus } \\
\text { agricoles }\end{array}$ & $\begin{array}{l}\text { Autres } \\
\text { revenus }\end{array}$ & Total revenu \\
\hline Cassou & Bûcherons & 46360 & 0 & 29512 & 17740 & $\mathbf{9 3 6 1 2}$ \\
\hline \multirow{3}{*}{ Silly-Pouni-Zawara } & Bûcherons & 106156 & 0 & 3333 & 8594 & $\mathbf{1 1 8 0 8 3}$ \\
\cline { 2 - 7 } & Charbonniers & 30303 & 178182 & 56063 & 727 & $\mathbf{2 6 5 4 0 6}$ \\
\cline { 2 - 7 } & Total moyen & $\mathbf{7 4 0 6 4}$ & $\mathbf{7 5 3 8 5}$ & $\mathbf{2 5 2 4 7}$ & $\mathbf{5 2 6 6}$ & $\mathbf{1 7 9} \mathbf{3 0 8}$ \\
\hline \multirow{3}{*}{ Sud Ouest Sissili } & Bûcherons & 82766 & 129574 & 112447 & 24223 & $\mathbf{3 4 9 0 1 1}$ \\
\cline { 2 - 7 } & Charbonniers & 160000 & 120000 & 0 & 0 & $\mathbf{2 8 0 0 0 0}$ \\
\cline { 2 - 7 } & Total moyen & $\mathbf{8 4 3 7 5}$ & $\mathbf{1 2 9 3 7 5}$ & $\mathbf{1 1 0 1 0 4}$ & $\mathbf{2 3 7 1 9}$ & $\mathbf{3 4 7 5 7 3}$ \\
\hline Sapoui Bieha & Bûcherons & 155715 & 0 & 0 & 15765 & $\mathbf{1 7 1 4 8 0}$ \\
\hline Nazinon & Bûcherons & 14324 & 500 & 2735 & 1412 & $\mathbf{1 8 9 7 1}$ \\
\hline Nakambé & Bûcherons & 149563 & 0 & 1406 & 0 & $\mathbf{1 5 0 9 6 9}$ \\
\hline Bougnounou & Bûcherons & 90000 & 0 & 3684 & 3368 & $\mathbf{9 7 0 5 3}$ \\
\hline \multirow{3}{*}{ Ensemble } & Bûcherons & 96187 & 22873 & 24160 & 10749 & $\mathbf{1 5 3 9 6 9}$ \\
\cline { 2 - 7 } & Charbonniers & 34118 & 176471 & 54364 & $\mathbf{7 0 6}$ & $\mathbf{2 6 5} \mathbf{8 4 8}$ \\
\cline { 2 - 7 } & Moyenne & $\mathbf{8 9 1 7 6}$ & $\mathbf{4 0 2 2 3}$ & $\mathbf{2 7 4 8 3}$ & $\mathbf{9 6 1 4}$ & $\mathbf{1 6 6 2 7 6}$ \\
\hline
\end{tabular}

Source: Analyse des données d'enquêtes bûcherons (mai-juin 2005).

Tableau 6 : Structure des revenus des bûcherons selon le village d'origine

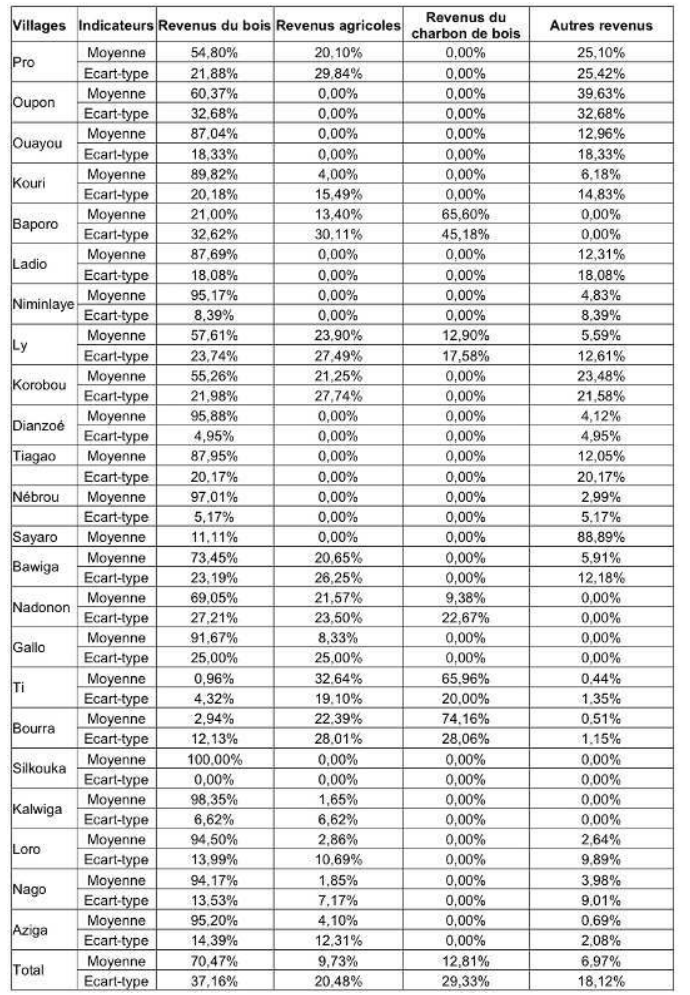

Source : Analyse des données d'enquêtes bûcherons (mai-juin 2005). 
Tableau 7. Revenus par chantier et par type d'activité des exploitants sondés en 2004

\begin{tabular}{l|l|l|l|l|l|l|}
\hline \multirow{2}{*}{ Zones forestières } & \multicolumn{6}{l|}{ Revenus totaux des exploitants forestiers par activités } \\
\cline { 2 - 7 } & Coupe du bois & Charbon de bois & Agricoles & Miel & Autres revenus & Total \\
\hline Lassou & 1159000 & 0 & 737800 & 0 & 443500 & 2340300 \\
\hline Silly-Pouni-Zawara & 5777000 & 5880000 & 1944000 & 0 & 410750 & 14011750 \\
\hline Sud Ouest Sissili & 4050000 & 6210000 & 5285000 & 85000 & 1053500 & 16683500 \\
\hline Sapoui Bieha & 7162900 & 0 & 0 & 0 & 725200 & 7888100 \\
\hline Vazinon & 487000 & 17000 & 93000 & 13000 & 35000 & 645000 \\
\hline Vakambé & 4786000 & 0 & 45000 & 0 & 0 & 4831000 \\
\hline Sougnounou & $\mathbf{3 4 2 0 0 0 0}$ & 0 & 140000 & 0 & 128000 & 3688000 \\
\hline Ensemble & $\mathbf{2 6 8 4 1 9 0 0}$ & $\mathbf{1 2 1 0 7 0 0 0}$ & $\mathbf{8 2 4 4 8 0 0}$ & $\mathbf{9 8 0 0 0}$ & $\mathbf{2 7 9 5 9 5 0}$ & $\mathbf{5 0 0 8 7 6 5 0}$ \\
\hline
\end{tabular}

Source : Analyse des données d'enquêtes bûcherons (mai-juin 2005).

Tableau 4 : Les indices FGT de pauvreté dans les villages riverains des 7 zones forestières

\begin{tabular}{|c|c|c|c|c|c|c|c|c|c|c|}
\hline \multirow{2}{*}{ Villages } & \multirow{2}{*}{$\mathrm{n}$} & \multirow{2}{*}{$q$} & \multirow{2}{*}{$A=\sum\left(\frac{Z-Y_{i}}{Z}\right)^{1}$} & \multirow{2}{*}{$B=\sum_{Y}^{2}\left(\frac{Z-Y_{i}}{Z}\right)^{2}$} & \multicolumn{2}{|c|}{ Incidence de pauvreté } & \multicolumn{2}{|c|}{$\begin{array}{c}\text { Profondeur de } \\
\text { pauvreté }\end{array}$} & \multicolumn{2}{|c|}{ Sévérité de pauvreté } \\
\hline & & & & & Indice & Contribution & Indice & Contribution & P2 & Contribution \\
\hline Pro & 13 & 7,00 & 2,83 & 1,26 & $53,85 \%$ & $6,25 \%$ & $21,75 \%$ & $5,35 \%$ & $9,70 \%$ & $3,63 \%$ \\
\hline Oupon & 10 & 6,00 & 4,02 & 2,80 & $60,00 \%$ & $5,36 \%$ & $40,16 \%$ & $7,59 \%$ & $28,02 \%$ & $8,08 \%$ \\
\hline Ouayou & 2 & 2,00 & 1,43 & 1,03 & $100,00 \%$ & $1,79 \%$ & $71,57 \%$ & $2,71 \%$ & $51,41 \%$ & $2,96 \%$ \\
\hline Kouri & 15 & 2,00 & 0,49 & 0,16 & $13,33 \%$ & $1,79 \%$ & $3,25 \%$ & $0,92 \%$ & $1,10 \%$ & $0,47 \%$ \\
\hline Baporo & 15 & 2,00 & 0,79 & 0,31 & $13,33 \%$ & $1,79 \%$ & $5,27 \%$ & $1,49 \%$ & $2,08 \%$ & $0,90 \%$ \\
\hline Ladio & 15 & 10,00 & 2,78 & 1,13 & $66,67 \%$ & $8,93 \%$ & $18,54 \%$ & $5,26 \%$ & $7,52 \%$ & $3,25 \%$ \\
\hline Niminlaye & 15 & 5,00 & 1,92 & 0,78 & $33,33 \%$ & $4,46 \%$ & $12,77 \%$ & $3,62 \%$ & $5,20 \%$ & $2,25 \%$ \\
\hline Ly & 13 & 1,00 & 0,09 & 0,01 & $0,00 \%$ & $0,00 \%$ & $0,00 \%$ & $0,00 \%$ & $0,00 \%$ & $0,00 \%$ \\
\hline Korobou & 15 & 2,00 & 0,67 & 0,23 & $13,33 \%$ & $1,79 \%$ & $4,46 \%$ & $1,27 \%$ & $1,54 \%$ & $0,67 \%$ \\
\hline Dianzoé & 15 & 2,00 & 0,79 & 0,32 & $13,33 \%$ & $1,79 \%$ & $5,27 \%$ & $1,49 \%$ & $2,13 \%$ & $0,92 \%$ \\
\hline Tiagao & 15 & 4,00 & 2,00 & 1,29 & $26,67 \%$ & $3,57 \%$ & $13,36 \%$ & $3,79 \%$ & $8,61 \%$ & $3,72 \%$ \\
\hline Nébrou & 15 & 1,00 & 0,00 & 0,00 & $6,67 \%$ & $0,89 \%$ & $0,01 \%$ & $0,00 \%$ & $0,00 \%$ & $0,00 \%$ \\
\hline Sayaro & 1 & 000 & 0,00 & 0,00 & $0,00 \%$ & $0,00 \%$ & $0,00 \%$ & $0,00 \%$ & $0,00 \%$ & $0,00 \%$ \\
\hline Bawiga & 13 & 13,00 & 9,12 & 7,78 & $100,00 \%$ & $11,61 \%$ & $70,13 \%$ & $17,24 \%$ & $59,88 \%$ & $22,44 \%$ \\
\hline Nadonon & 12 & 12,00 & 9,79 & 8,28 & $100,00 \%$ & $10,71 \%$ & $81,55 \%$ & $18,51 \%$ & $69,02 \%$ & $23,87 \%$ \\
\hline Gallo & 9 & 9,00 & 7,29 & 6,06 & $100,00 \%$ & $8,04 \%$ & $81,05 \%$ & $13,79 \%$ & $67,31 \%$ & $17,46 \%$ \\
\hline $\mathrm{Ti}$ & 20 & 0,00 & 0,00 & 0,00 & $0,00 \%$ & $0,00 \%$ & $0,00 \%$ & $0,00 \%$ & $0,00 \%$ & $0,00 \%$ \\
\hline Bourra & 17 & 0,00 & 0,00 & 0,00 & $0,00 \%$ & $0,00 \%$ & $0,00 \%$ & $0,00 \%$ & $0,00 \%$ & $0,00 \%$ \\
\hline Silkouka & 16 & 5,00 & 1,11 & 0,28 & $31,25 \%$ & $4,46 \%$ & $6,91 \%$ & $2,09 \%$ & $1,73 \%$ & $0,80 \%$ \\
\hline Kalwiga & 16 & 3,00 & 1,06 & 0,42 & $18,75 \%$ & $2,68 \%$ & $6,65 \%$ & $2,01 \%$ & $2,61 \%$ & $1,20 \%$ \\
\hline Loro & 14 & 11,00 & 3,38 & 1,30 & $78,57 \%$ & $9,82 \%$ & $24,14 \%$ & $6,39 \%$ & $9,32 \%$ & $3,76 \%$ \\
\hline Nago & 15 & 8,00 & 2,01 & 0,86 & $53,33 \%$ & $7,14 \%$ & $13,42 \%$ & $3,81 \%$ & $5,76 \%$ & $2,49 \%$ \\
\hline Aziga & 9 & 7,00 & 1,31 & 0,38 & $77,78 \%$ & $6,25 \%$ & $14,61 \%$ & $2,49 \%$ & $4,20 \%$ & $1,09 \%$ \\
\hline Total & 300 & 112,00 & 52,88 & 34,70 & $37,33 \%$ & $17,63 \%$ & $11,57 \%$ & $37,33 \%$ & $17,63 \%$ & $11,57 \%$ \\
\hline
\end{tabular}

Source : Analyse des données d'enquêtes bûcherons (mai-juin 2005).

Tableau 5 :Indices de pauvreté dans les chantiers forestiers aménagés

\begin{tabular}{|l|l|l|l|l|l|l|}
\hline & \multicolumn{2}{|l|}{ Incidence de pauvreté } & \multicolumn{2}{l|}{ Profondeur de pauvreté } & \multicolumn{2}{l|}{ Sévérité de pauvreté } \\
\hline Zone forestière & Indice & Contribution & Indice & Contribution & Indice & Contribution \\
\hline Cassou & 0,60 & $13,39 \%$ & 0,33 & $15,65 \%$ & 0,20 & $14,67 \%$ \\
\hline Silly-Pouni-Zawara & 0,25 & $16,96 \%$ & 0,08 & $11,30 \%$ & 0,03 & $6,88 \%$ \\
\hline Sud Ouest sissili & 0,06 & $2,68 \%$ & 0,02 & $1,44 \%$ & 0,01 & $0,69 \%$ \\
\hline Sapoui Bieha & 0,15 & $6,25 \%$ & 0,06 & $5,29 \%$ & 0,04 & $4,65 \%$ \\
\hline Nazinon & 1,00 & $30,36 \%$ & 0,77 & $49,54 \%$ & 0,65 & $63,77 \%$ \\
\hline Nakambé & 0,25 & $7,14 \%$ & 0,07 & $4,10 \%$ & 0,02 & $2,00 \%$ \\
\hline Bougnounou & 0,68 & $23,21 \%$ & 0,18 & $12,68 \%$ & 0,07 & $7,34 \%$ \\
\hline Ensemble & $\mathbf{0 , 3 7}$ & $\mathbf{3 7 , 3 3 \%}$ & $\mathbf{0 , 1 8}$ & $\mathbf{1 7 , 6 3 \%}$ & $\mathbf{0 , 1 2}$ & $\mathbf{1 1 , 5 7 \%}$ \\
\hline
\end{tabular}

Source : Analyse des données d'enquêtes bûcherons (mai-juin 2005). 


\section{NOTES}

1. Site Web de la FAO 2006: http://www.fao.org/forestry

\section{RÉSUMÉS}

Cet article utilise des données d'enquêtes sur les 8 principaux chantiers forestiers aménagés pour l'exploitation du bois-énergie au Burkina Faso, pour analyser l'impact de cette politique forestière sur les conditions de vie des ménages riverains de ces zones forestières. Ces enquêtes qui ont été financées par USAID à travers l'Antenne Régionale du Centre International de Recherche en Foresterie (CIFOR) en mai-juin 2005, ont couvert 355 bûcherons de 24 villages situés entre $35 \mathrm{~km}$ et $250 \mathrm{~km}$ de la ville de Ouagadougou. Une première approche de la structure des revenus des bûcherons a révélé la prédominance des revenus issus de l'exploitation forestière sur les autres sources de revenus de ceux-ci, et aussi la contribution de cette activité à l'amélioration des conditions de vie des bûcherons. Les méthodes des percentiles et des indices de pauvreté ont été utilisées pour appréhender l'incidence, la profondeur et la sévérité de pauvreté parmi les bûcherons exploitant ces zones forestières. Il en ressort que l'incidence de pauvreté des bûcherons est inférieure à celle évaluée au plan national en 2003. Il en est de même pour la profondeur de la pauvreté dans ces zones forestières qui s'est révélée être inférieure à celle obtenue au niveau national. Cependant, la sévérité moyenne de pauvreté sur ces zones forestières observées est supérieure à celle observée au niveau national bien qu'il existence une forte disparité de ce seuil entre zones forestières et villages de ces zones.

This article uses data from a large survey funded by US-AID and implemented by the Center for International Forestry Research (CIFOR) on the main eight managed forest areas for wood fuel production in Burkina Faso (may-june, 2005), for analyzing the effects of this forest management policy on peasants living conditions around these forest areas. This survey has covered 355 woodcutters of 24 villages located between $35 \mathrm{~km}$ and $250 \mathrm{~km}$ from Ouagadougou the capital city of this country. A preliminary approach of woodcutters' income structure has revealed the magnitude of wood fuel income on their other income sources, and also the great contribution of this activity on the improvement of woodcutters' living conditions. Percentiles and poverty indexes' methods have been used to capture poverty incidence, deepness and severity among woodcutters of these managed forest areas. It worked out that woodcutters' poverty prevalence (incidence) in these forest areas is lower than that computed at national level in 2003. It's the same for the poverty deepness index which also is lower than that found at the national level. Although, the average poverty severity index on these observed forest areas has been greater than that observed in 2003 at the national level even if it exists a high dispersion of this index among forest areas and villages of these forest areas. 


\section{AUTEUR}

\section{BOUKARY OUEDRAOGO}

Boukary Ouedraogo est Maître-Assistant, membre du Laboratoire d'Economie de l'Environnement et de Socioéconométrie du CEDRES de l'UFR/SEG de l'UO et chercheur associé du GREThA de l'université Montesquieu Bordeaux IV (France) docteur à l'Université de Ouagadougou, Burkina Faso ; boukary.ouedraogo@univ-ouaga.bf 